\title{
Prioritizing Data/Energy Thresholding-Based Antenna Switching for SWIPT-Enabled Secondary Receiver in Cognitive Radio Networks
}

\author{
Fatma Benkhelifa, Student Member, IEEE, and Mohamed-Slim Alouini, Fellow, IEEE
}

\begin{abstract}
Simultaneous wireless power and information transfer (SWIPT) is considered in cognitive radio networks with a multi-antenna energy harvesting (EH) secondary receiver (SR). The SR harvests the energy from the secondary transmitter and primary transmitter. The SR uses the antenna switching technique which selects a subset of antennas to decode the information (namely the information decoding (ID) antennas) and the rest to harvest the energy (namely the EH antennas). The AS technique is performed via a thresholding-based strategy inspired from the maximum ratio combining technique with an output threshold (OT-MRC) which is proposed in two ways: the prioritizing data selection (PDS) scheme, and the prioritizing energy selection (PES) scheme. For both schemes, we study the expressions and the asymptotic results of the probability mass function of the selected ID antennas, the average harvested energy, the power outage probability, and the data outage probability. We deduce the performance of the joint PDS and PES scheme. We evaluate all performance metrics for the Rayleigh and Nakagami fading channels. Through the simulation results, we show the impact of different simulation parameters on the performance metrics. We also show that there is a tradeoff between the data and energy performance metrics.
\end{abstract}

Index Terms-Energy harvesting, Simultaneous Wireless Information and Power Transfer (SWIPT), cognitive radio (CR), spectrum sharing (SS), antenna switching (AS), maximum ratio combining (MRC), probability mass function (PMF), average harvested energy, power outage probability, data outage probability, prioritizing data/energy selection schemes.

\section{INTRODUCTION}

Energy consumption and spectrum scarcity are two major issues in wireless communication systems. For the next generation of wireless communication systems, it is challenging to assure the energy efficiency as well the spectrum efficiency. On the one hand, cognitive radio (CR) networks are a promising solution to solve the spectrum scarcity. CR networks allow the unlicensed users to use the spectrum whenever the licensed users are idle [1]. For the spectrum sharing (SS) in CR networks, the unlicensed users and licensed users are allowed to share the spectrum as long as the interference induced by the unlicensed users do not harm the licensed users [2]. Various works have studied the spectrum sharing in CR networks with single/multiple antennas at the primary and secondary

F. Benkhelifa and M.-S. Alouini are with the Division of Computer, Electrical, and Mathematical Sciences \& Engineering (CEMSE), King Abdullah University of Science and Technology (KAUST), Thuwal, Makkah Province, Saudi Arabia. [e-mail: \{fatma.benkhelifa, slim.alouini\}@kaust.edu.sa].

This paper was accepted in part at IEEE International Conference on Communications (ICC'2017), Paris, France. networks [3]-[7]. The secondary capacity gains were investigated for the SS in single-input single-output (SISO) CR networks with imperfect channel state information (CSI) in [3]. The overlay SS in multiple-input multiple-output (MIMO) CR networks was studied in [4] where exact expressions of the rate and bit error rate (BER) were derived, as well as the asymptotic analysis at high signal-to-noise ratio (SNR). In [5], a ratio selection scheme was proposed to select one antenna to transmit at the secondary receiver (SR) using a mean value (MV)-based power allocation strategy. For that, closedform expressions and asymptotic results of outage probability, symbol error rate (SER) and ergodic capacity were derived for the secondary network using MV-based scheme, as well as CSI-based scheme. In [6], the capacity of SS in CR networks with the maximum ratio combining (MRC) technique was investigated when SR has multiple antennas and undergoes asymmetric fading. In [7], an opportunistic spectrum sharing in a CR network was proposed to maximize the downlink throughput where few cognitive users are scheduled for transmission out of a large number of multi-antenna secondary users.

On the other hand, energy harvesting is a promising solution to make the wireless communication systems more energy efficient and self-sustainable. Among the various energy sources, the radio frequency (RF) signals are found to be a good source for energy harvesting. The process of the simultaneous use of RF signals for energy transfer and information transfer is known in the literature as the simultaneous wireless power and information transfer (SWIPT). The SWIPT technique was first studied in SISO communication systems in [8], [9] where flat fading and frequency selective channels were considered and a tradeoff between the information rate and the energy transfer was shown for the co-located information decoding (ID) and energy harvesting (EH) receivers. Then, SWIPT for MIMO communication systems was considered in [10] where practical EH schemes were proposed which separate the operation of the ID and EH receivers over the power domain or the time domain. The proposed practical schemes are termed as the power splitting (PS) and the time switching (TS). Following that, more research interest to the application of SWIPT in wireless systems was conducted, in particular SWIPT in relay communication systems [11]-[14]. In [11], an amplify-and-forward (AF) SISO relay communication system was considered where the relay is an EH node that harvests the energy from the source and uses the harvested energy to forward the source signal to the destination. 
Two relaying protocols were considered: PS-based relaying and TS-based relaying. For both relaying protocols, analytic expressions of the outage probability and ergodic capacity were derived for delay-limited and delay-tolerant transmission modes, respectively. In [12], a MIMO decode-and-forward relay communication system was considered where the relay is an EH multi-antennas node using the antenna switching technique which spatially separates the operation of EH and ID receivers. Indeed, the AS technique in [12] allocates the strongest antennas to decode the information data while the others to harvest the energy (or vice versa). In [13], [14], the source/relay precoding design was considered to maximize the achievable end-to-end rate in AF and DF MIMO relay systems, respectively. The relay is an EH constrained node that uses fully/partially the harvested energy to forward the source signal to the destination. Possibly imperfect CSI was considered in both works. In [15], the distributed uplink user association problem in EH ultra-dense small cell networks (UD-SCNs) was solved using an approach based on the meanfield multi-armed bandit games. In [16], the ambient RF-based EH (UD-SCNs) was considered where the implementation feasibility and the tradeoffs in terms of the energy efficiency and data outage probability of a typical user were investigated. In [17], using the online ski rental framework, the on/off scheduling problem of self-powered small cell base stations (SBSs) was considered which aims to minimize the operational energy consumption and transmission delay costs.

Subsequently, it is interesting to investigate the SWIPT technique in cognitive radio networks. Recently, various researchers have shown their interest to study the SWIPT technique in CR networks [18]-[25]. In [18], an opportunistic spectrum access scheme was considered in CR networks where secondary transmitters (STs) either harvest energy from ambient transmissions or transmit signals when primary transmitters (PTs) are far away. In [19], the closed form expression and the high SNR approximation of the outage probability were derived for underlay $\mathrm{CR}$ networks with one primary receiver (PR), one cognitive transmitter-receiver, and one EH relay. In [20]-[22], CR relay networks were considered where the ST or the secondary relay assists the primary transmission while harvesting the energy using the PS or TS scheme. In [20],the joint optimization of the power splitter factor and the energy allocation over $N$ time slots which maximizes the throughput was considered and a suboptimal management algorithm was proposed in an $\mathrm{AF}$ relay cognitive network with one primary receiver, one cognitive transmitter-receiver, and one EH relay. The EH relay harvests the energy from the received signals using the PS scheme. In [21], the primary and secondary data outage probability and the rate-energy tradeoff between the maximum ergodic capacity and the maximum harvested energy in the secondary network were analyzed in an AF cognitive relay network where the ST and the SR have energy harvesting capabilities using the PS scheme. Moreover, ST acts as a relay for the primary transmission, harvests the energy from the primary signal, and forwards the primary signal and transmits to its receiver simultaneously. In [22], the optimal cooperation strategy, the time allocation and the power allocation were investigated in non-cooperation and cooperation modes to maximize the secondary user's achievable throughout in a CR network system where the ST acts as a DF basedrelay for the primary transmission. The ST is self-powered and harvests the energy from the ambient transmitters using the save-then-transmit protocol. In [23], [24], an underlay CR network is studied where the ST is self-powered and harvests the energy from the primary transmission. In [23], the online optimal time allocation between the EH phase and ID phase is proposed which maximizes the average achievable rate of the cognitive radio system, subject to the $\epsilon$-percentile protection criteria for the primary system where the ST harvests the energy from the primary transmission. In [24], a channel quality based threshold and opportunistic scheduling were exploited in CR networks with one ST and multiple EH SRs under the peak interference power constraint of the PR and the ST maximum transmit power limit. Each SR is scheduled to harvest energy if the channel condition is above the threshold or to decode information if the channel condition is below the threshold. In this context, the analytical expressions of the secondary ergodic capacity, SER, throughput rate, and energy harvesting were investigated. In [25], the mutual outage probability (MOP) was investigated for SS in CR networks with SWIPT-enabled multi-antenna PR where a win-to-win situation was shown at both PR and SR sides.

In line with the research scope, we propose to investigate the SWIPT for spectrum sharing in CR networks where the multi-antenna EH SR harvests the energy from the primary and secondary transmission using the antenna switching technique. The antenna switching technique at SR is performed based on a thresholding technique inspired from the MRC technique with an output threshold (OT-MRC). The OT-MRC combining technique was studied before in [26] where the co-channel interference (CCI) was not considered. In this paper, we propose two antenna selection schemes employing a thresholding technique based on OT-MRC with CCI: one scheme is prioritizing the information data and the second is prioritizing the harvested energy. The prioritizing data selection (PDS) scheme selects the ID antennas at SR where the received power from ST is above a certain threshold $\gamma_{t h}$, while the prioritizing energy selection (PES) scheme selects the EH antennas at SR where the received power from ST is above $\gamma_{t h}$. Also, we deduce the performance of the joint PDS and PES scheme. For the Rayleigh fading channels, we derive the expressions and asymptotic results of the probability mass function (PMF) of the selected number of antennas connected to the ID circuits at SR, the average harvested energy, the power outage probability, and the data outage probability, for both PDS and PES selection schemes and the joint PDS and PES scheme. For the Nakgami fading channels, we derive the expressions and asymptotic results of the probability mass function (PMF) of the selected number of antennas connected to the ID circuits at SR, the average harvested energy, and the data outage probability, for both PDS and PES selection schemes and we deduce the case of scalability at ST and PT. Through the simulation results, we show the energy and data performance of both schemes and the accuracy of the derived analytic results compared to Monte Carlo simulations. Also, we observe the impact of different 


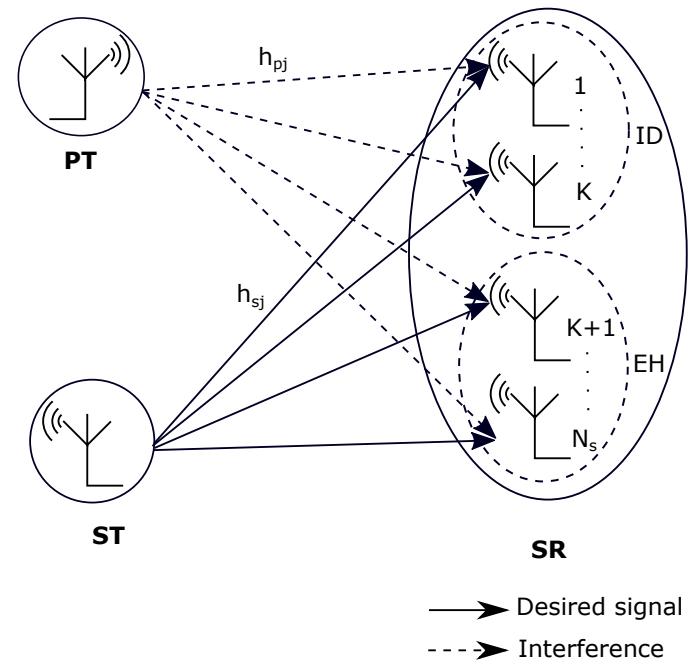

Figure 1: CR Network System with SWIPT-Enabled Secondary Receiver

simulation parameters on the derived performance metrics and we show that there is an energy-data tradeoff between the data performance metric which is the data outage probability and the energy performance metrics which are the average harvested energy and the power outage probability for the two schemes.

\section{System Model}

We consider a cognitive network consisting of a primary transmitter PT, a primary receiver PR, a secondary transmitter ST and a secondary receiver SR. While the PT, PR and ST are battery powered, the SR is self-powered by its harvested energy from the RF signals sent from ST and PT. All the nodes are equipped with single antennas, except the SR which is equipped with multiple antennas $N_{s}$. The channel between the PT and the $j$ 'th antenna of SR, and the channel between the ST and the $j$ 'th antenna of SR are denoted by $h_{p j}$, and $h_{s j}$, respectively, $\forall j=1, \ldots, N_{s}$. All the channels $h_{p j}$, and $h_{s j}$ are independent identically distributed (i.i.d.).

\section{A. SWIPT-Enabled Secondary Receiver}

The SR harvests energy using the antenna switching (AS) technique. In fact, the AS technique assigns a subset of the multiple antennas to harvest energy and the remaining to decode the received data information. Let $K$ be the number of antennas connected to the ID circuits and $N_{s}-K$ be the number of antennas connected to the EH circuits, with $K \leq N_{s}$. How to choose $K$ will be discussed later.

For a given $K$, the combined signal-to-interference ratio (SIR) at the ID antennas at SR is given by

$$
\gamma_{I D}(K)=\frac{P_{s}}{P_{p}} \frac{\sum_{j=1}^{K}\left|h_{s j}\right|^{2}}{\sum_{j=1}^{K}\left|h_{p j}\right|^{2}}=\frac{\Gamma_{s}(K)}{\Gamma_{p}(K)},
$$

where $P_{s}$ is the transmit power at ST, $P_{p}$ is the transmit power at PT, and $\Gamma_{s}(K)=P_{s} \sum_{j=1}^{K}\left|h_{s j}\right|^{2}$ and $\Gamma_{p}(K)=P_{p} \sum_{j=1}^{K}\left|h_{p j}\right|^{2}$ are the received power at the ID antennas from ST and PT, respectively.
The harvested energy at the EH antennas at SR is given by

$$
Q(K)= \begin{cases}\zeta\left(\bar{\Gamma}_{s}(K)+\bar{\Gamma}_{p}(K)\right), & \text { if } 0 \leq K<N_{s}, \\ 0, & \text { if } K=N_{s},\end{cases}
$$

where $\zeta$ is the conversion efficiency and $\bar{\Gamma}_{s}(K)=$ $P_{s} \sum_{K+1}^{N_{s}}\left|h_{s j}\right|^{2}$ and $\bar{\Gamma}_{p}(K)=P_{p} \sum_{K+1}^{N_{s}}\left|h_{p j}\right|^{2}$ are the received power at the EH antennas from ST and PT, respectively.

Now, the question to ask is how to choose $K$. For that, we present different selection schemes based on a thresholding technique inspired from the OT-MRC technique studied in [26]. The proposed selection schemes are presented in the following section.

\section{B. Performance Metrics}

In order to evaluate the performance of the considered selection schemes, we propose to study the performance metrics such as the PMF $P_{K}(k)$ of $K$, the expected value of the harvested energy, the power outage probability, and the data outage probability.

- The expected value of the harvested energy is defined as

$$
\bar{Q}=\mathbb{E}[Q]=\sum_{k=1}^{N_{s}-1} \mathbb{E}[Q(k) \mid K=k] P_{K}(k),
$$

where $\mathbb{E}[\cdot]$ is the mean operator.

- Moreover, the power outage probability is defined as:

$$
\mathcal{P O}\left(\gamma_{q}\right)=\operatorname{Prob}\left(Q \leq \gamma_{q}\right)=\sum_{k=1}^{N_{s}-1} \operatorname{Prob}\left(Q(k) \leq \gamma_{q} \& K=k\right),
$$

where $\gamma_{q}$ is referring to the minimum required harvested power at SR, and $\operatorname{Prob}(\cdot)$ is the probability density function of a random variable. If the selection of $K$ is independent of $Q(K)$, then

$$
\mathcal{P O}\left(\gamma_{q}\right)=\sum_{k=1}^{N_{s}-1} \operatorname{Prob}\left(Q(k) \leq \gamma_{q} \mid K=k\right) P_{K}(k) .
$$

- In addition, the data outage probability at SR is defined

$P_{\text {out }}\left(\xi_{s}\right)=\operatorname{Prob}\left(\gamma_{I D}<\xi_{s}\right)=\sum_{k=1}^{N_{s}-1} \operatorname{Prob}\left(\gamma_{I D}(k)<\xi_{s} \& K=k\right)$,

where $\xi_{s}=2^{R}-1$ and $R$ is the transmission rate at ST. If the selection of $K$ is independent of $\gamma_{I D}(K)$, then

$$
P_{\text {out }}\left(\xi_{s}\right)=\sum_{k=1}^{N_{s}-1} \operatorname{Prob}\left(\gamma_{I D}(k)<\xi_{s} \mid K=k\right) P_{K}(k) .
$$

\section{Proposed Thresholding-Based Antenna Selection SCHEMES}

In this section, we present the proposed thresholding based selection schemes and we evaluate their performance.

In order to select the number of antennas $K$, we use a thresholding technique inspired from the OT-MRC technique in the presence of the co-channel interference (CCI) from PT. Note that the OT-MRC technique was previously considered only in the no CCI case [26]. In order to derive the OT-MRC technique with CCI, we will follow the same steps in [26]. 
Let $\Gamma_{G}(K)$ be the combined utility function to be specified depending the selection scheme considered. With OT-MRC, the number of antennas $K$ is selected so that the chosen combined utility function $\Gamma_{G}(K)$ above a certain predefined threshold. Starting from the single-antenna case, the OT-MRC combiner gradually raises the number of antennas in a way to raise $\Gamma_{G}(K)$ above the threshold $\gamma_{t h}$. Please note the antennas are randomly ordered. For more details about OT-MRC, please refer to [26]. In what follows, we consider two selection schemes based on different combined utility functions.

- Prioritizing data selection (PDS) scheme:

- $\Gamma_{G}(K)$ stands for the received power at ID circuits at $\mathrm{SR}$ from the desired transmitter ST, i.e. $\Gamma_{G}(K)=$ $\Gamma_{s}(K)$.

- $K$ corresponds to the case where $\Gamma_{s}(K)$ is greater than the threshold $\gamma_{t h}$.

- Prioritizing energy selection (PES) scheme:

- $\Gamma_{G}(K)$ stands for the received power at EH circuits at SR (proportional to the harvested energy) from the desired transmitter ST, i.e. $\Gamma_{G}(K)=\bar{\Gamma}_{s}(K)$.

- $K$ corresponds to the case where $\bar{\Gamma}_{s}(K)$ is greater than the threshold $\gamma_{t h}$.

Evaluating the PDS ans PES schemes, we deduce the performance of the joint PDS and PES scheme that selects the minimum of the number of antennas selected by the PDS and PES schemes.

\section{A. Prioritizing Data Selection (PDS) Scheme}

The prioritizing data selection PDS scheme selects the antennas $K$ in a way to assure that the received power from the desired transmitter ST at the information decoding receivers at SR is above a certain predefined threshold. Here, the utility combined function stands for the received power at ID circuits at SR from the desired transmitter ST, i.e. $\Gamma_{G}(K)=\Gamma_{s}(K)$. The selected number of antennas $K$ corresponds to the case where $\Gamma_{s}(K)$ is greater than the threshold $\gamma_{t h}$. Let us denote by $\gamma_{s, j}=P_{s}\left|h_{s j}\right|^{2}$, for $j=1, \ldots, N_{s}$. For the PDS scheme, the thresholding procedure is as follows:

- Start with $j=1$ and $\Gamma_{s}(1)=\gamma_{s, 1}$.

- If $\Gamma_{s}(j)<\gamma_{t h}$, update $\Gamma_{s}(j+1)=\Gamma_{s}(j)+\gamma_{s, j+1}$ and $j=j+1$.

- Repeat until $\Gamma_{s}(j) \geq \gamma_{\text {th }}$ or $j \geq N_{s}-1$.

At the end, $K$ will be equal to $j$.

Remark 1: Note that the selected number of antennas $K$ cannot be equal to zero or to $N_{s}$ in a way to avoid the two worse cases when all the receiving antennas are used for harvesting energy and no antennas are used for decoding information ( $K=0$, i.e. no data) and when all the receiving antennas are used for decoding information and no antennas are used for harvesting energy ( $K=N_{s}$, i.e. no energy). Same remark holds for the PES scheme.

1) $P M F$ of $K$ :

Based on the selection PDS scheme, the PMF of $K$ is given by

$P_{K}^{(1)}(k)= \begin{cases}\operatorname{Prob}\left(\Gamma_{s}(1) \geq \gamma_{t h}\right), & \text { if } k=1, \\ \operatorname{Prob}\left(\Gamma_{s}(k-1)<\gamma_{t h} \leq \Gamma_{s}(k)\right), & \text { if } k=2, \ldots, N_{s}-2, \\ \operatorname{Prob}\left(\Gamma_{s}\left(N_{s}-2\right)<\gamma_{t h}\right), & \text { if } k=N_{s}-1,\end{cases}$ which is equivalently written as

$P_{K}^{(1)}(k)= \begin{cases}1-F_{\gamma_{s}}^{(1)}\left(\gamma_{t h}\right), & \text { if } k=1, \\ F_{\gamma_{s}}^{(k-1)}\left(\gamma_{t h}\right) & \\ -\int_{0}^{\gamma_{t h}} f_{\gamma_{s}}^{(1)}(z) F_{\gamma_{s}}^{(k-1)}\left(\gamma_{t h}-z\right) \mathrm{d} z, & \text { if } k=2, \ldots, N_{s}-2, \\ F_{\gamma_{s}}^{\left(N_{s}-2\right)}\left(\gamma_{t h}\right), & \text { if } k=N_{s}-1,\end{cases}$

where $f_{\gamma_{s}}^{(k)}(\cdot)$ and $F_{\gamma_{s}}^{(k)}(\cdot)$ are the probability density function (PDF) and cumulative distribution function $(\mathrm{CDF})$ of $\Gamma_{s}(k)$, respectively, for $k=1, \ldots, N_{s}-1$.

2) Average of Harvested Energy:

The average harvested energy at SR is given by

$$
\begin{aligned}
\bar{Q}^{(1)} & =\zeta \sum_{k=1}^{N_{s}-1} \sum_{j=k+1}^{N_{s}}\left(P_{s} \mathbb{E}\left[\left|h_{s j}\right|^{2}\right]+P_{p} \mathbb{E}\left[\left|h_{p j}\right|^{2}\right]\right) P_{K}^{(1)}(k) \\
& =\zeta \sum_{k=1}^{N_{s}-1}\left(N_{s}-k\right)\left(P_{s} \mathbb{E}\left[\left|h_{s j}\right|^{2}\right]+P_{p} \mathbb{E}\left[\left|h_{p j}\right|^{2}\right]\right) P_{K}^{(1)}(k) .
\end{aligned}
$$

\section{3) Power Outage Probability:}

Since the choice of $K$ is independent of the harvested energy, we can write that the power outage probability is given by

$$
\mathcal{P} \boldsymbol{O}^{(1)}\left(\gamma_{q}\right)=\sum_{k=1}^{N_{s}-1} F_{Q^{(1)}(k)}\left(\gamma_{q}\right) P_{K}^{(1)}(k)
$$

where $F_{Q^{(1)}(k)}(\cdot)$ is the CDF of $Q^{(1)}(k)$ which was shown in Appendix A to be given by

$$
F_{Q^{(1)}(k)}\left(\gamma_{q}\right)=\int_{0}^{\frac{\gamma_{q}}{\zeta}} f_{\gamma_{s}}^{\left(N_{s}-k\right)}(x) F_{\gamma_{p}}^{\left(N_{s}-k\right)}\left(\frac{\gamma_{q}}{\zeta}-x\right) \mathrm{d} x,
$$

where $F_{\gamma_{p}}^{(k)}(\cdot)$ is the $\mathrm{CDF}$ of $\Gamma_{p}(k)$, for $k=1, \ldots, N_{s}-1$. Hence, we deduce

$$
\mathcal{P} O^{(1)}\left(\gamma_{q}\right)=\sum_{k=1}^{N_{s}-1}\left[\int_{0}^{\frac{\gamma_{q}}{\zeta}} f_{\gamma_{s}}^{\left(N_{s}-k\right)}(x) F_{\gamma_{p}}^{\left(N_{s}-k\right)}\left(\frac{\gamma_{q}}{\zeta}-x\right) \mathrm{d} x\right] P_{K}^{(1)}(k) \text {. }
$$

\section{4) Data Outage Probability:}

The secondary data outage probability is written as

$$
P_{\text {out }}^{(1)}\left(\xi_{s}\right)=\operatorname{Prob}\left(\frac{\Gamma_{s}(k)}{\Gamma_{p}(k)}<\xi_{s} \& K=k\right)=\sum_{k=1}^{N_{s}-1} \mathcal{I}_{k}\left(\xi_{s}, \gamma_{t h}\right),
$$

where $I_{1}\left(\xi_{s}, \gamma_{t h}\right), I_{k}\left(\xi_{s}, \gamma_{t h}\right)$, for $k=2, \ldots, N_{s}-2$, and $I_{N_{s}-1}\left(\xi_{s}, \gamma_{t h}\right)$ were shown in Appendix B to be expressed as in (76), (78), and (81). Consequently, the secondary data outage probability is given by $(16)$, where $f_{\gamma_{p}}^{(k)}(\cdot)$ is the PDF of $\Gamma_{p}(k)$, for $k=1, \ldots, N_{s}-1$.

\section{B. Prioritizing Energy Selection (PES) Scheme}

By contrast to the prioritizing data selection PDS scheme, the prioritizing energy selection (PES) scheme selects the antennas $K$ in a way to assure that the received power from the desired transmitter ST at the EH receivers at SR is above a certain predefined threshold. Hence, the utility combined , function stands for the received power at $\mathrm{EH}$ circuits at SR (proportional to the harvested energy) from the desired transmitter ST, i.e. $\Gamma_{G}(K)=\bar{\Gamma}_{s}(K)$. The selected number of 


$$
\begin{aligned}
P_{\text {out }}^{(1)}\left(\xi_{s}\right) & =\int_{\frac{\gamma_{t h}}{\xi_{s}}}^{\infty} F_{\gamma_{s}}^{(1)}\left(\xi_{s} y\right) f_{\gamma_{p}}^{(1)}(y) \mathrm{d} y-F_{\gamma_{s}}^{(1)}\left(\gamma_{t h}\right)\left[1-F_{\gamma_{p}}^{(1)}\left(\frac{\gamma_{t h}}{\xi_{s}}\right)\right]+\int_{0}^{\frac{\gamma_{t h}}{\xi_{s}}}\left[\int_{0}^{\xi_{s} y} F_{\gamma_{s}}^{\left(N_{s}-2\right)}\left(-z+\xi_{s} y\right) f_{\gamma_{s}}^{(1)}(z) \mathrm{d} z\right] f_{\gamma_{p}}^{\left(N_{s}-1\right)}(y) \mathrm{d} y \\
& +\sum_{k=2}^{N_{s}-1} \int_{\frac{\gamma_{t h}}{\xi_{s}}}^{\infty}\left[\int_{\xi_{s} y-\gamma_{t h}}^{\xi_{s} y} F_{\gamma_{s}}^{(k-1)}\left(-z+\xi_{s} y\right) f_{\gamma_{s}}^{(1)}(z) \mathrm{d} z\right] f_{\gamma_{p}}^{(k)}(y) \mathrm{d} y-\sum_{k=2}^{N_{s}-2}\left[1-F_{\gamma_{p}}^{(k)}\left(\frac{\gamma_{t h}}{\xi_{s}}\right)\right] \int_{0}^{\gamma_{t h}} F_{\gamma_{s}}^{(k-1)}\left(\gamma_{t h}-z\right) f_{\gamma_{s}}^{(1)}(z) \mathrm{d} z \\
& +\sum_{k=2}^{N_{s}-1} F_{\gamma_{s}}^{(k-1)}\left(\gamma_{t h}\right) \int_{\frac{\gamma_{t h}}{\xi_{s}}}^{\infty} F_{\gamma_{s}}^{(1)}\left(\xi_{s} y-\gamma_{t h}\right) f_{\gamma_{p}}^{(k)}(y) \mathrm{d} y .
\end{aligned}
$$

antennas $K$ corresponds to the case where $\bar{\Gamma}_{s}(K)$ is greater than the threshold $\gamma_{t h}$. For the PES scheme, the thresholding procedure is as follows:

- Start with $j=N_{s}-1$ and $\bar{\Gamma}_{s}\left(N_{s}-1\right)=\gamma_{N_{s}}$.

- If $\bar{\Gamma}_{s}(j)<\gamma_{t h}$, update $j=j-1$ and $\bar{\Gamma}_{s}(j)=\bar{\Gamma}_{s}(j+1)+\gamma_{s, j+1}$.

- Repeat until $\bar{\Gamma}_{s}(j) \geq \gamma_{t h}$ or $j \leq 1$.

At the end, $K$ will be equal to $j$.

1) $P M F$ of $K$ :

The PMF of $K$ is given by:

$P_{K}^{(2)}(k)= \begin{cases}\operatorname{Prob}\left(\bar{\Gamma}_{s}(2)<\gamma_{t h}\right), & \text { if } k=1, \\ \operatorname{Prob}\left(\bar{\Gamma}_{s}(k+1)<\gamma_{t h} \leq \bar{\Gamma}_{s}(k)\right), & \text { if } k=2, \ldots, N_{s}-2, \\ \operatorname{Prob}\left(\bar{\Gamma}_{s}\left(N_{s}-1\right) \geq \gamma_{t h}\right), & \text { if } k=N_{s}-1,\end{cases}$

which is equivalently written as

$$
P_{K}^{(2)}(k)= \begin{cases}F_{\gamma_{s}}^{\left(N_{s}-2\right)}\left(\gamma_{t h}\right), & \text { if } k=1, \\ F_{\gamma_{s}}^{\left(N_{s}-k-1\right)}\left(\gamma_{t h}\right)-\int_{0}^{\gamma_{t h}} f_{\gamma_{s}}^{(1)}(z) & \text { if } k=2, \ldots, N_{s}-2, \\ \times F_{\gamma_{s}}^{\left(N_{s}-k-1\right)}\left(\gamma_{t h}-z\right) \mathrm{d} z, & \text { if } k=N_{s}-1 . \\ 1-F_{\gamma_{s}}^{(1)}\left(\gamma_{t h}\right), & \end{cases}
$$

Remark 2: We note that $P_{K}^{(2)}(k)=P_{K}^{(1)}\left(N_{s}-k\right)$ if the PDS and PES schemes use the same predefined threshold $\gamma_{t h}$.

2) Average of Harvested Energy:

The average harvested energy at SR is given by

$$
\bar{Q}^{(2)}=\zeta \sum_{k=1}^{N_{s}-1}\left(N_{s}-k\right)\left(P_{s} \mathbb{E}\left[\left|h_{s j}\right|^{2}\right]+P_{p} \mathbb{E}\left[\left|h_{p j}\right|^{2}\right]\right) P_{K}^{(2)}(k)
$$

Remark 3: Note that we have $\bar{Q}^{(2)}=\zeta\left(\varepsilon_{s}+\varepsilon_{p}\right) N_{s}-\bar{Q}^{(1)}$, if the condition $P_{K}^{(2)}(k)=P_{K}^{(1)}\left(N_{s}-k\right)$ is satisfied.

3) Power Outage Probability:

The power outage probability is written as

$$
\begin{aligned}
\mathcal{P O}^{(2)}\left(\gamma_{q}\right) & =\sum_{k=1}^{N_{s}-1} \operatorname{Prob}\left(\bar{\Gamma}_{s}(k)+\bar{\Gamma}_{p}(k) \leq \frac{\gamma_{q}}{\zeta} \& K=k\right) \\
& =\sum_{k=1}^{N_{s}-1} \mathcal{J}_{k}\left(\gamma_{q}, \gamma_{t h}\right),
\end{aligned}
$$

where $\mathcal{J}_{1}\left(\gamma_{q}, \gamma_{t h}\right), \mathcal{J}_{k}\left(\gamma_{q}, \gamma_{t h}\right)$, for $k=2, \ldots, N_{s}-2$, and $\mathcal{J}_{N_{s}-1}\left(\gamma_{q}, \gamma_{t h}\right)$ are expressed in Appendix $\mathrm{C}$ depending on the values of $\delta_{q, t h}=\frac{\gamma_{q}}{\zeta}-\gamma_{t h}$ and $\gamma_{t h}$, as in (85), (95), and (105), respectively. Subsequently, the power outage probability is expressed, depending on the values of $\delta_{q, t h}$ and $\gamma_{t h}$, as $\mathcal{P O}(2)\left(\gamma_{q}\right)$

$$
= \begin{cases}\mathcal{G}_{0}, & \text { if } \delta_{q, t h} \leq 0, \\ \sum_{k=1}^{N_{s}-1} \mathcal{G}_{1}(k)+\sum_{k=2}^{N_{s}-2} \mathcal{G}_{2}\left(k, \delta_{q, t h}, \frac{\gamma_{q}}{\zeta}\right), & \text { if } 0<\delta_{q, t h}<\gamma_{t h}, \\ \sum_{k=1}^{N_{s}-1} \mathcal{G}_{1}(k)+\sum_{k=2}^{N_{s}-2} \mathcal{G}_{2}\left(k, \gamma_{t h}, \delta_{q, t h}\right), & \text { if } \gamma_{t h} \leq \delta_{q, t h},\end{cases}
$$

where $\mathcal{G}_{0}, \mathcal{G}_{1}(k)$, and $\mathcal{G}_{2}(k, x, y)$ are defined in (86), (87), and (96), respectively.

4) Data Outage Probability:

The secondary data outage probability is given by

$$
\begin{aligned}
P_{\text {out }}^{(2)}\left(\xi_{s}\right) & =\sum_{k=1}^{N_{s}-1} \operatorname{Prob}\left(\frac{\Gamma_{s}(k)}{\Gamma_{p}(k)}<\xi_{s} \mid K=k\right) P_{K}^{(2)}(k) \\
& =\sum_{k=1}^{N_{s}-1}\left[\int_{0}^{\infty} f_{\gamma_{p}}^{(k)}(y) F_{\gamma_{s}}^{(k)}\left(\xi_{s} y\right) \mathrm{d} y\right] P_{K}^{(2)}(k) .
\end{aligned}
$$

\section{Joint PDS and PES Scheme}

In this part, we propose to study a selection scheme combining both PDS and PES schemes where the selected number of antennas $K$ is the minimum of the one chosen by the PDS scheme and the one chosen by the PES scheme. Let $K^{(3)}$ be the number of antennas chosen by the PDS and PES schemes combined where $K^{(3)}$ verifies

$$
K^{(3)}=\min \left(K^{(1)}, K^{(2)}\right),
$$

where $K^{(1)}$ and $K^{(2)}$ are the number of ID antennas chosen by the PDS scheme, and the PES scheme, respectively. In this case, the PMF of $K^{(3)}$ is expressed as

$$
\begin{aligned}
& P_{K}^{(3)}(k) \\
& = \begin{cases}P_{K}^{(1)}(1)+P_{K}^{(2)}(1)\left(1-P_{K}^{(1)}(1)\right), & \text { if } k=1, \\
P_{K}^{(1)}(k) \sum_{j=k}^{N_{s}-1} P_{K}^{(2)}(j) & \text { if } k=2, \ldots, N_{s}-2, \\
+P_{K}^{(2)}(k) \sum_{j=k+1}^{N_{s}-1} P_{K}^{(1)}(j), & \text { if } k=N_{s}-1 . \\
P_{K}^{(1)}\left(N_{s}-1\right) P_{K}^{(2)}\left(N_{s}-1\right), & \end{cases}
\end{aligned}
$$

Subsequently, the average harvested energy is given by

$$
\bar{Q}^{(3)}=\zeta\left(\varepsilon_{s}+\varepsilon_{p}\right)\left[N_{s}-\sum_{k=1}^{N_{s}-1} P_{K}^{(3)}(k)\right]
$$

In addition, the data outage probability is expressed as

$$
\begin{aligned}
P_{\text {out }}^{(3)}\left(\xi_{s}\right) & =\sum_{k=1}^{N_{s}-1} \mathcal{I}_{k}\left(\xi_{s}, \gamma_{t h}\right)\left(1-F_{\gamma_{s}}^{\left(N_{s}-k\right)}\left(\gamma_{t h}\right)\right) \\
& +\sum_{k=1}^{N_{s}-1}\left[\int_{0}^{\frac{\gamma_{t h}}{\xi_{s}}} f_{\gamma_{p}}^{(k)}(y) F_{\gamma_{s}}^{(k)}\left(\xi_{s} y\right) \mathrm{d} y\right] P_{K}^{(2)}(k) \\
& +\sum_{k=1}^{N_{s}-1} F_{\gamma_{s}}^{(k)}\left(\gamma_{t h}\right)\left[1-F_{\gamma_{p}}^{(k)}\left(\frac{\gamma_{t h}}{\xi_{s}}\right)\right] P_{K}^{(2)}(k) .
\end{aligned}
$$


Furthermore, the power outage probability is expressed as

$$
\begin{aligned}
\mathcal{P} O^{(3)}\left(\gamma_{q}\right) & =\sum_{k=1}^{N_{s}-1}\left[\int_{0}^{\frac{\gamma_{q}}{\zeta}-\gamma_{t h}} f_{\gamma_{s}}^{\left(N_{s}-k\right)}(x) F_{\gamma_{p}}^{\left(N_{s}-k\right)}\left(\frac{\gamma_{q}}{\zeta}-x\right) \mathrm{d} x\right] P_{K}^{(1)}(k) \\
& -\sum_{k=1}^{N_{s}-1} F_{\gamma_{p}}^{\left(N_{s}-k\right)}\left(\frac{\gamma_{q}}{\zeta}-\gamma_{t h}\right) F_{\gamma_{s}}^{\left(N_{s}-k\right)}\left(\gamma_{t h}\right) P_{K}^{(1)}(k) \\
& +\sum_{k=1}^{N_{s}-1} \mathcal{J}_{k}\left(\gamma_{q}, \gamma_{t h}\right) F_{\gamma_{s}}^{(k)}\left(\gamma_{t h}\right)
\end{aligned}
$$

\section{Example: Rayleigh Fading Special Case}

Let us assume that all the channels $h_{p j}$, and $h_{s j}$ are modeled as flat fading with Rayleigh distribution with variances $\lambda_{p s}$, and $\lambda_{s s}$, respectively. Let us denote by $\varepsilon_{s}=P_{s} \lambda_{s s}, \varepsilon_{p}=P_{p} \lambda_{p s}$, and $\varepsilon_{s p}=-\frac{1}{\varepsilon_{s}}+\frac{1}{\varepsilon_{p}}$.

\section{A. PDS Scheme}

\section{1) $P M F$ of $K$ :}

The PMF of $K$ in (9) can be written as

$$
P_{K}^{(1)}(k)= \begin{cases}\frac{1}{\Gamma(k)}\left(\frac{\gamma_{t h}}{\varepsilon_{s}}\right)^{k-1} e^{-\frac{\gamma_{t h}}{\varepsilon_{s}}}, & \text { if } k=1, \ldots, N_{s}-2, \\ \frac{\gamma\left(N_{s}-2, \frac{y_{k}}{\varepsilon_{s}}\right)}{\Gamma\left(N_{s}-2\right)}, & \text { if } k=N_{s}-1,\end{cases}
$$

which was obtained using Table I-(a), where $\Gamma(\cdot)$ and $\gamma(\cdot, \cdot)$ are the Gamma function and the lower incomplete Gamma function [27], respectively.

2) Average of Harvested Energy:

The average harvested energy in (11) at SR can be written as

$$
\begin{aligned}
\bar{Q}^{(1)} & =\frac{\zeta\left(\varepsilon_{s}+\varepsilon_{p}\right)}{\Gamma\left(N_{s}-2\right)}\left[\left(N_{s}-1-\frac{\gamma_{t h}}{\varepsilon_{s}}\right) \Gamma\left(N_{s}-2, \frac{\gamma_{t h}}{\varepsilon_{s}}\right)\right. \\
& \left.+e^{-\frac{\gamma_{t h}}{\varepsilon_{s}}}\left(\frac{\gamma_{t h}}{\varepsilon_{s}}\right)^{N_{s}-2}+\gamma\left(N_{s}-2, \frac{\gamma_{t h}}{\varepsilon_{s}}\right)\right],
\end{aligned}
$$

which was obtained using $\sum_{m=0}^{s-1} \frac{x^{m}}{\Gamma(m+1)}=\frac{e^{x} \Gamma(s, x)}{\Gamma(s)}$ in [27, (8.352.4)] and the corresponding derivative $\sum_{m=0}^{s-1} \frac{m x^{m-1}}{\Gamma(m+1)}=$ $\frac{e^{x} \Gamma(s, x)}{\Gamma(s)}-\frac{x^{s-1}}{\Gamma(s)}$, where $\Gamma(\cdot, \cdot)$ is the upper incomplete gamma function [27].

\section{3) Power Outage Probability:}

Using Table I-(b), the power outage probability in (14) can be shown equal to (32) depending on the value of $\varepsilon_{s p}$ either zero or not, where ${ }_{1} F_{1}(a ; b ; z)$ is the Kummer confluent hypergeometric function [27].

4) Data Outage Probability:

In Appendix B, we have shown that $\mathcal{I}_{1}\left(\xi_{s}, \gamma_{t h}\right), \mathcal{I}_{k}\left(\xi_{s}, \gamma_{t h}\right)$ and $\mathcal{I}_{N_{s}-1}\left(\xi_{s}, \gamma_{t h}\right)$ are given by (77), (80) and (83), respectively. Consequently, the secondary data outage probability is given by (33).

5) Remarks and Asymptotic Results:

- When $\frac{\gamma_{t h}}{\varepsilon_{s}} \rightarrow 0$, only one antenna is selected to decode the information data and we have

$$
\begin{aligned}
& \bar{Q}^{(1)} \approx\left(N_{s}-1\right) \zeta\left(\varepsilon_{s}+\varepsilon_{p}\right), \\
& \mathcal{P} O^{(1)}\left(\gamma_{q}\right) \approx \begin{cases}\frac{\gamma\left(2\left(N_{s}-1\right), \frac{\gamma_{q}}{\zeta \varepsilon_{s}}\right)}{\Gamma\left(2\left(N_{s}\right)\right.}, & \text { if } \varepsilon_{s p}=0, \\
\frac{\gamma\left(N_{s}-1, \frac{\gamma_{q}}{\zeta \varepsilon_{s}}\right)}{\Gamma\left(N_{s}-1\right)}-\left(\frac{\gamma_{q}}{\zeta \varepsilon_{s}}\right)^{N_{s}-1} e^{-\frac{\gamma q}{\zeta \varepsilon_{p}}} & \\
\times \sum_{j=0}^{N_{s}-2} \frac{\left(\frac{\gamma_{q}}{\zeta \varepsilon_{p}}\right)^{j} F_{1}\left(N_{s}-1 ; N_{s}+j ; \frac{\gamma_{q}}{\zeta} \varepsilon_{s p}\right)}{\Gamma\left(N_{s}+j\right)}, & \text { if } \varepsilon_{s p} \neq 0,\end{cases}
\end{aligned}
$$

$$
P_{\text {out }}^{(1)}\left(\xi_{s}\right) \approx \frac{\xi_{s} \varepsilon_{p}}{\xi_{s} \varepsilon_{p}+\varepsilon_{s}} e^{-\frac{\gamma_{t h}}{\xi_{s} p_{p}}} .
$$

- When $\frac{\gamma_{t h}}{\varepsilon_{s}} \rightarrow \infty$, only one antenna is selected to harvest the energy and we have

$$
\begin{aligned}
\bar{Q}^{(1)} & \approx \zeta\left(\varepsilon_{s}+\varepsilon_{p}\right), \\
\mathcal{P} O^{(1)}\left(\gamma_{q}\right) & \approx \begin{cases}1-\left(1+\frac{\gamma_{q}}{\zeta \varepsilon_{s}}\right) e^{-\frac{\gamma_{q}}{\zeta s_{s}}}, & \text { if } \varepsilon_{s p}=0, \\
1-\frac{\varepsilon_{s}-\frac{\gamma_{q}}{\zeta \varepsilon_{s}}-\varepsilon_{p} e^{-\frac{\gamma_{q}}{\zeta \varepsilon_{p}}}}{\varepsilon_{s}-\varepsilon_{p}}, & \text { if } \varepsilon_{s p} \neq 0,\end{cases} \\
P_{\text {out }}^{(1)}\left(\xi_{s}\right) & \approx \frac{\Gamma\left(N_{s}-1, \frac{\gamma_{t h}}{\xi_{s} \varepsilon_{p}}\right)}{\Gamma\left(N_{s}-1\right)} .
\end{aligned}
$$

\section{B. PES Scheme}

1) $P M F$ of $K$ :

Similarly to $P_{K}^{(1)}(k)$, we can show that

$$
P_{K}^{(2)}(k)= \begin{cases}\frac{\gamma\left(N_{s}-2, \frac{\gamma_{t h}}{\varepsilon_{s}}\right)}{\Gamma\left(N_{s}-2\right)}, & \text { if } k=1, \\ \frac{1}{\Gamma\left(N_{s}-k\right)}\left(\frac{\gamma_{t h}}{\varepsilon_{s}}\right)^{N_{s}-k-1} e^{-\frac{\gamma_{t h}}{\varepsilon_{s}}}, & \text { if } k=2, \ldots, N_{s}-1 .\end{cases}
$$

\section{2) Average of Harvested Energy:}

The average harvested energy at SR can be written as

$$
\begin{aligned}
\bar{Q}^{(2)} & =\frac{\zeta\left(\varepsilon_{s}+\varepsilon_{p}\right)}{\Gamma\left(N_{s}-2\right)}\left[\left(N_{s}-1\right) \gamma\left(N_{s}-2, \frac{\gamma_{t h}}{\varepsilon_{s}}\right)\right. \\
& \left.+\left(1+\frac{\gamma_{t h}}{\varepsilon_{s}}\right) \Gamma\left(N_{s}-2, \frac{\gamma_{t h}}{\varepsilon_{s}}\right)-e^{-\frac{\gamma_{t h}}{\varepsilon_{s}}}\left(\frac{\gamma_{t h}}{\varepsilon_{s}}\right)^{N_{s}-2}\right],
\end{aligned}
$$

which was obtained in a similar way as (31).

3) Power Outage Probability:

In Appendix C, we have shown that $\mathcal{J}_{1}\left(\gamma_{q}, \gamma_{t h}\right), \mathcal{J}_{k}\left(\gamma_{q}, \gamma_{t h}\right)$ and $\mathcal{J}_{N_{s}-1}\left(\gamma_{q}, \gamma_{t h}\right)$ are expressed depending on $\delta_{q, \text { th }}$ and $\varepsilon_{s p}$ in (89) and (91), in (98) and (102), and in (106), respectively. Consequently, the power outage probability is given by (42) and (43), depending on $\varepsilon_{s p}$, where $\psi(k, x)=\frac{\gamma\left(2\left(N_{s}-k\right), x\right)}{\Gamma\left(2\left(N_{s}-k\right)\right)}, \varphi(k, x)=\frac{\gamma\left(N_{s}-k, x\right)}{\Gamma\left(N_{s}-k\right)}$, $\chi(k, x)=\left(\frac{x}{\varepsilon_{p}}\right)^{N_{s}-k} e^{-\frac{x}{\varepsilon_{s}}} \sum_{j=0}^{N_{s}-k-1} \frac{\left(\frac{x}{\varepsilon_{s}}\right)_{1}^{j} F_{1}\left(N_{s}-k ; N_{s}-k+j+1 ;-\varepsilon_{s p} x\right)}{\Gamma\left(N_{s}-k+j+1\right)}$, $\Phi_{1}\left(k, \gamma_{t h}, \delta_{q, t h}\right)$, and $\Omega_{1}\left(k, \gamma_{t h}, \delta_{q, t h}\right)$ are given in (92) and in (93), for $k=1, \ldots, N_{s}-1$, respectively, $\Phi_{2}\left(k, \gamma_{t h}, \delta_{q, t h}\right)$, $\Omega_{2}\left(k, \gamma_{t h}, \delta_{q, t h}\right)$, and $\Omega_{3}\left(k, \gamma_{t h}, \delta_{q, t h}\right)$ are given in (99), in (100) and in (103), for $k=2, \ldots, N_{s}-2$, respectively.

4) Data Outage Probability:

For the Rayleigh fading channels, we can write

$$
\begin{aligned}
P_{\text {out }}^{(2)}\left(\xi_{s}\right) & =\frac{\xi_{s} \varepsilon_{p}}{\varepsilon_{s}+\xi_{s} \varepsilon_{p}} \frac{\gamma\left(N_{s}-2, \frac{\gamma_{t h}}{\varepsilon_{s}}\right)}{\Gamma\left(N_{s}-2\right)}+e^{-\frac{\gamma_{t h}}{\varepsilon_{s}}} \sum_{k=2}^{N_{s}-1} \frac{\Gamma(2 k)}{\Gamma(k)^{2} k \Gamma\left(N_{s}-k\right)} \\
& \times \frac{\left(\xi_{s} \varepsilon_{p} \varepsilon_{s}\right)^{k}\left(\frac{\gamma_{t h}}{\varepsilon_{s}}\right)^{N_{s}-k-1}{ }_{2} F_{1}\left(1,2 k ; k+1 ; \frac{\xi_{s} \varepsilon_{p}}{\varepsilon_{s}+\xi_{s} \varepsilon_{p}}\right)}{\left(\varepsilon_{s}+\xi_{s} \varepsilon_{p}\right)^{2 k}}
\end{aligned}
$$

where (44) was obtained using $[27, \quad(6.455 .2)]$, and ${ }_{2} F_{1}(a, b ; c ; z)$ is the Gaussian hypergeometric function.

5) Remarks and Asymptotic Results:

- When $\frac{\gamma_{t h}}{\varepsilon_{s}} \rightarrow 0$, only one antenna is selected to harvest the energy and we have

$$
\bar{Q}^{(2)} \approx \zeta\left(\varepsilon_{s}+\varepsilon_{p}\right)
$$




$$
\begin{aligned}
& \mathcal{P} \boldsymbol{O}^{(1)}\left(\gamma_{q}\right)
\end{aligned}
$$

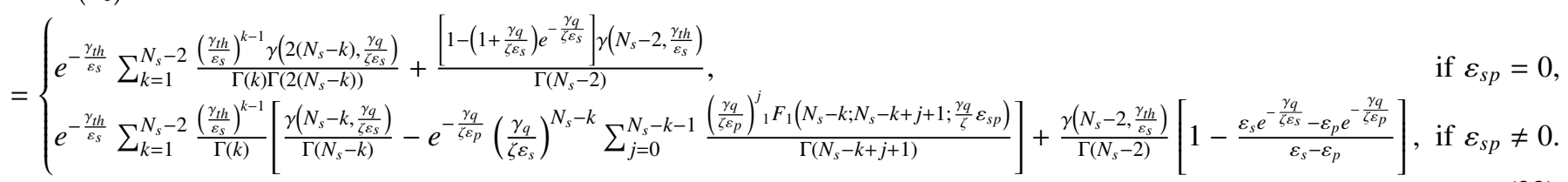

$$
\begin{aligned}
P_{\text {out }}^{(1)}\left(\xi_{s}\right)= & \frac{\xi_{s} \varepsilon_{p} e^{-\gamma_{t h}\left(\frac{1}{\varepsilon_{s}}+\frac{1}{\xi_{s}}\right)}}{\xi_{s} \varepsilon_{p}+\varepsilon_{s}}-\sum_{k=2}^{N_{s}-2} \frac{\left(\frac{\gamma_{t h}}{\varepsilon_{s}}\right)^{k-1} e^{-\frac{\gamma_{t h}}{\varepsilon_{s}}}}{\Gamma(k)^{2}}\left[\frac{e^{\frac{\gamma_{t h}}{\varepsilon_{s}}} \Gamma\left(k, \gamma_{t h}\left(\frac{1}{\varepsilon_{s}}+\frac{1}{\xi_{s} \varepsilon_{p}}\right)\right)}{\left(\xi_{s} \frac{\varepsilon_{p}}{\varepsilon_{s}}+1\right)^{k}}-\Gamma\left(k, \frac{\gamma_{t h}}{\xi_{s} \varepsilon_{p}}\right)\right]+1-\frac{\Gamma\left(N_{s}-2, \frac{\gamma_{t h}}{\varepsilon_{s}}\right) \Gamma\left(N_{s}-1, \frac{\gamma_{t h}}{\xi_{s} \varepsilon_{p}}\right)}{\Gamma\left(N_{s}-2\right) \Gamma\left(N_{s}-1\right)} \\
& -\frac{\left(\frac{\gamma_{t h}}{\varepsilon_{s}}\right)^{N_{s}-2} \Gamma\left(N_{s}-1, \gamma_{t h}\left(\frac{1}{\xi_{s} \varepsilon_{p}}+\frac{1}{\varepsilon_{s}}\right)\right)}{\Gamma\left(N_{s}-1\right)^{2} \varepsilon_{p}^{N_{s}-1}\left(\frac{1}{\varepsilon_{p}}+\frac{\xi_{s}}{\varepsilon_{s}}\right)^{N_{s}-1}}-\frac{1}{\Gamma\left(N_{s}-1\right)} \sum_{m=0}^{N_{s}-2} \frac{\left(\frac{\xi_{s} \varepsilon_{p}}{\varepsilon_{s}}\right)^{m} \gamma\left(N_{s}+m-1, \gamma_{t h}\left(\frac{1}{\varepsilon_{s}}+\frac{1}{\xi_{s} \varepsilon_{p}}\right)\right)}{\Gamma(m+1)\left(\frac{\xi_{s} \varepsilon_{p}}{\varepsilon_{s}}+1\right)^{N_{s}+m-1}}
\end{aligned}
$$

- If $\varepsilon_{s p}=0$,

$$
\mathcal{P} O^{(2)}\left(\gamma_{q}\right)= \begin{cases}\psi\left(1, \frac{\gamma_{q}}{\zeta \varepsilon_{s}}\right), & \text { if } \delta_{q, t h} \leq 0, \\ \sum_{k=1}^{N_{s}-1} \Phi_{1}\left(k, \gamma_{t h}, \delta_{q, t h}\right)+\sum_{k=2}^{N_{s}-2} \Phi_{2}\left(k, \gamma_{t h}, \delta_{q, t h}\right), & \text { if } 0<\delta_{q, t h}<\gamma_{t h}, \\ \sum_{k=1}^{N_{s}-1} \Phi_{1}\left(k, \gamma_{t h}, \delta_{q, t h}\right), & \text { if } \gamma_{t h} \leq \delta_{q, t h} .\end{cases}
$$

- If $\varepsilon_{s p} \neq 0$,

$$
\mathcal{P} O^{(2)}\left(\gamma_{q}\right)= \begin{cases}\varphi\left(1, \frac{\gamma_{q}}{\zeta \varepsilon_{p}}\right)-\chi\left(1, \frac{\gamma_{q}}{\zeta}\right), & \text { if } \delta_{q, t h} \leq 0, \\ \sum_{k=1}^{N_{s}-1} \Omega_{1}\left(k, \gamma_{t h}, \delta_{q, t h}\right)+\sum_{k=2}^{N_{s}-2} \Omega_{2}\left(k, \gamma_{t h}, \delta_{q, t h}\right), & \text { if } 0<\delta_{q, t h}<\gamma_{t h}, \\ \sum_{k=1}^{N_{s}-1} \Omega_{1}\left(k, \gamma_{t h}, \delta_{q, t h}\right)+\sum_{k=2}^{N_{s}-2} \Omega_{3}\left(k, \gamma_{t h}, \delta_{q, t h}\right), & \text { if } \gamma_{t h} \leq \delta_{q, t h} .\end{cases}
$$

$$
\begin{aligned}
& P_{\text {out }}^{(2)}\left(\xi_{s}\right) \approx \frac{\Gamma\left(2\left(N_{s}-1\right)\right)\left(\xi_{s} \varepsilon_{p} \varepsilon_{s}\right)^{N_{s}-1}}{\Gamma\left(N_{s}-1\right)^{2}\left(N_{s}-1\right)\left(\varepsilon_{s}+\xi_{s} \varepsilon_{p}\right)^{2\left(N_{s}-1\right)}} \\
& \times{ }_{2} F_{1}\left(1,2\left(N_{s}-1\right) ; N_{s} ; \frac{\xi_{s} \varepsilon_{p}}{\varepsilon_{s}+\xi_{s} \varepsilon_{p}}\right) \text {, } \\
& \mathcal{P} \boldsymbol{O}^{(2)}\left(\gamma_{q}\right) \\
& \approx\left\{\begin{array}{l}
1-\left(1+\frac{\gamma_{q}}{\zeta \varepsilon_{s}}\right) e^{-\frac{\gamma_{q}}{\zeta \varepsilon_{s}}}, \text { if } \varepsilon_{s p}=0 \& \delta_{q, t h}>0, \\
1-\frac{\varepsilon_{s} e^{-\frac{\gamma_{q}}{\zeta \varepsilon_{s}}}-\varepsilon_{p} e^{-\frac{\delta_{q, t h}}{\varepsilon_{p}}}}{\varepsilon_{s}-\varepsilon_{p}}, \text { if } \varepsilon_{s p} \neq 0 \& \delta_{q, t h}>0 .
\end{array}\right.
\end{aligned}
$$

- When $\frac{\gamma_{t h}}{\varepsilon_{s}} \rightarrow \infty$, only one antenna is selected to decode the information data and we have

$$
\begin{aligned}
\overline{\mathbf{Q}}^{(2)} & \approx\left(N_{s}-1\right) \zeta\left(\varepsilon_{s}+\varepsilon_{p}\right), \\
P_{\text {out }}^{(2)}\left(\xi_{s}\right) & \approx \frac{\xi_{s} \varepsilon_{p}}{\varepsilon_{s}+\xi_{s} \varepsilon_{p}}, \\
\mathcal{P} O^{(2)}\left(\gamma_{q}\right) & \approx \mathcal{J}_{1}\left(x, \gamma_{t h}\right) .
\end{aligned}
$$

\section{Joint PDS and PES Scheme}

For the Rayleigh fading channels, the PMF of $K^{(3)}$ can be shown equal to

$$
P_{K}^{(3)}(k)=
$$

$$
\begin{cases}e^{-\frac{\gamma_{t h}}{\varepsilon_{s}}}+\left(1-e^{\left.-\frac{\gamma_{t h}}{\varepsilon_{s}}\right)} \frac{\gamma\left(N_{s}-2, \frac{\gamma_{t h}}{\varepsilon_{s}}\right)}{\Gamma\left(N_{s}-2\right)},\right. & \text { if } k=1, \\ e^{-\frac{\gamma_{t h}}{\varepsilon_{s}}}\left[\frac{\left(\frac{\gamma_{t h}}{\varepsilon_{s}}\right)^{k-1}}{\Gamma(k)} \frac{\Gamma\left(N_{s}-k, \frac{\gamma_{t h}}{\varepsilon_{s}}\right)}{\Gamma\left(N_{s}-k\right)}+\frac{\left(\frac{\gamma_{h}}{\varepsilon_{s}}\right)^{N_{s}-k-1}}{\Gamma\left(N_{s}-k\right)}\right. & \\ \left.\times \frac{\Gamma\left(N_{s}-1, \frac{\gamma_{t h}}{\varepsilon_{s}}\right)}{\Gamma\left(N_{s}-1\right)}-\frac{\left(\frac{\gamma_{h}}{\varepsilon_{s}}\right)^{N_{s}-k-1}}{\Gamma\left(N_{s}-k\right)} \frac{\Gamma\left(k, \frac{\gamma_{t h}}{\varepsilon_{s}}\right)}{\Gamma(k)}\right], & \text { if } k=2, \ldots, N_{s}-2, \\ e^{-\frac{\gamma_{t h}}{\varepsilon_{s}}} \frac{\gamma\left(N_{s}-2, \frac{\gamma_{t h}}{\varepsilon_{s}}\right)}{\Gamma\left(N_{s}-2\right)}, & \text { if } k=N_{s}-1 .\end{cases}
$$

Subsequently, the average harvested energy is chosen to be equal to

$$
\begin{aligned}
\bar{Q}^{(3)} & =\zeta\left(\varepsilon_{s}+\varepsilon_{p}\right)\left[\left(1-e^{-\frac{\gamma_{t h}}{\varepsilon_{s}}}\right)\left(N_{s}-1\right)+\frac{\Gamma\left(N_{s}-2, \frac{\gamma_{t h}}{\varepsilon_{s}}\right)}{\Gamma\left(N_{s}-2\right)}\right. \\
& \times\left(1+\left(N_{s}-2\right) e^{-\frac{\gamma_{t h}}{\varepsilon_{s}}}-\left(N_{s}-1-\frac{\gamma_{t h}}{\varepsilon_{s}}\right) \frac{\Gamma\left(N_{s}-1, \frac{\gamma_{t h}}{\varepsilon_{s}}\right)}{\Gamma\left(N_{s}-1\right)}\right) \\
& -\left(\frac{\left(\frac{\gamma_{t h}}{\varepsilon_{s}}\right)^{N_{s}-2}}{\Gamma\left(N_{s}-2\right)}-\left(N_{s}-1\right)\right) e^{-\frac{\gamma_{t h}}{\varepsilon_{s}}} \frac{\Gamma\left(N_{s}-1, \frac{\gamma_{t h}}{\varepsilon_{s}}\right)}{\Gamma\left(N_{s}-1\right)} \\
& \left.-e^{-\frac{\gamma_{t h}}{\varepsilon_{s}}} \sum_{n=2}^{N_{s}-2}\left(N_{s}-2 n\right) \frac{\left(\frac{\gamma_{t h}}{\varepsilon_{s}}\right)^{N_{s}-n-1}}{\Gamma\left(N_{s}-n\right)} \frac{\Gamma\left(n, \frac{\gamma_{t h}}{\varepsilon_{s}}\right)}{\Gamma(n)}\right] .
\end{aligned}
$$

In addition, the data outage probability is given by

$$
P_{\text {out }}^{(3)}\left(\xi_{s}\right)=\sum_{k=1}^{N_{s}-1} \frac{\mathcal{I}_{k}\left(\xi_{s}, \gamma_{t h}\right) \Gamma\left(N_{s}-k, \frac{\gamma_{t h}}{\varepsilon_{s}}\right)}{\Gamma\left(N_{s}-k\right)}-\frac{\gamma\left(N_{s}-2, \frac{\gamma_{t h}}{\varepsilon_{s}}\right)}{\Gamma\left(N_{s}-1\right) \varepsilon_{p}}
$$




$$
\begin{aligned}
& \times\left[\frac{1-e^{-\frac{\gamma_{t h}}{\xi_{s}}\left(\frac{1}{\varepsilon_{p}}+\frac{\xi_{s}}{\varepsilon_{s}}\right)}}{\frac{1}{\varepsilon_{p}}+\frac{\xi_{s}}{\varepsilon_{s}}}\right]+\left[1-e^{-\frac{\gamma_{t h}}{\xi_{s}}\left(\frac{1}{\varepsilon_{p}}+\frac{\xi_{s}}{\varepsilon_{s}}\right)}\right] \frac{\gamma\left(N_{s}-2, \frac{\gamma_{t h}}{\varepsilon_{s}}\right)}{\Gamma\left(N_{s}-2\right)} \\
& -\sum_{k=2}^{N_{s}-1} \frac{\left(\frac{\gamma_{t h}}{\varepsilon_{s}}\right)^{N_{s}-k-1} e^{-\frac{\gamma_{t h}}{\varepsilon_{s}}}}{\Gamma\left(N_{s}-k\right) \Gamma(k) \varepsilon_{p}^{k}}\left[\sum_{j=0}^{k-1} \frac{\left(\frac{\xi_{s}}{\varepsilon_{s}}\right)^{j} \gamma\left(k+j, \frac{\gamma_{t h}}{\xi_{s}}\left(\frac{1}{\varepsilon_{p}}+\frac{\xi_{s}}{\varepsilon_{s}}\right)\right)}{\Gamma(j+1)\left(\frac{1}{\varepsilon_{p}}+\frac{\xi_{s}}{\varepsilon_{s}}\right)^{k+j}}\right] \\
& +\sum_{k=2}^{N_{s}-1} \frac{\left(\frac{\gamma_{t h}}{\varepsilon_{s}}\right)^{N_{s}-k-1} e^{-\frac{\gamma_{t h}}{\varepsilon_{s}}}}{\Gamma\left(N_{s}-k\right)}\left[\frac{\gamma\left(k, \frac{\gamma_{t h}}{\xi_{s} \varepsilon_{p}}\right)}{\Gamma(k)}+\frac{\gamma\left(k, \frac{\gamma_{t h}}{\varepsilon_{s}}\right) \Gamma\left(k, \frac{\gamma_{t h}}{\xi_{s} \varepsilon_{p}}\right)}{\Gamma(k)^{2}}\right] .
\end{aligned}
$$

Furthermore, the power outage probability is written in (54) and (55) depending on the difference between $\varepsilon_{s}$ and $\varepsilon_{p}$ either zero or not.

\section{Example: Nakagami Fading Special Case}

In this section, we assume that the channels $h_{s j}$, and $h_{p j}$ are modeled as flat fading with Nakagami distribution with parameters $m_{1}$ and $\bar{\lambda}_{s s}$ and with parameters $m_{2}$ and $\bar{\lambda}_{p s}$, respectively. In other words, the PDF and $\mathrm{CDF}$ of $\Gamma_{s}(k)$, and $\Gamma_{p}(k)$ are given by [26]

$$
\begin{aligned}
& f_{\gamma_{s}}^{(k)}(x)=\left(\frac{m_{1}}{\varepsilon_{s}}\right)^{k m_{1}} \frac{x^{k m_{1}-1}}{\Gamma\left(k m_{1}\right)} e^{-\frac{m_{1}}{\varepsilon_{s}} x}, \\
& F_{\gamma_{s}}^{(k)}(x)=1-\frac{\Gamma\left(k m_{1}, \frac{m_{1}}{\varepsilon_{s}} x\right)}{\Gamma\left(k m_{1}\right)}, \\
& f_{\gamma_{p}}^{(k)}(x)=\left(\frac{m_{2}}{\varepsilon_{p}}\right)^{k m_{2}} \frac{x^{k m_{2}-1}}{\Gamma\left(k m_{2}\right)} e^{-\frac{m_{2}}{\varepsilon_{p}} x}, \\
& F_{\gamma_{p}}^{(k)}(x)=1-\frac{\Gamma\left(k m_{2}, \frac{m_{2}}{\varepsilon_{p}} x\right)}{\Gamma\left(k m_{2}\right)},
\end{aligned}
$$

with $\varepsilon_{s}=P_{s} \bar{\lambda}_{s s}$ and $\varepsilon_{p}=P_{p} \bar{\lambda}_{p s}$.

\section{A. PDS Scheme}

\section{1) $P M F$ of $K$ :}

We use the expression in Table I-(f) to show that the PMF of $K$ for the PDS scheme is given by

$$
P_{K}^{(1)}(k)= \begin{cases}\frac{\Gamma\left(m_{1}, \frac{m_{1}}{s_{s}} \gamma_{t h}\right)}{\Gamma\left(m_{1}\right)}, & \text { if } k=1, \\ \frac{\Gamma\left(k m_{1}, \frac{m_{1}}{\varepsilon_{s}} \gamma_{t h}\right)}{\Gamma\left(k m_{1}\right)}-\frac{\Gamma\left((k-1) m_{1}, \frac{m_{1}}{\varepsilon_{s}} \gamma_{t h}\right)}{\Gamma\left((k-1) m_{1}\right)}, & \text { if } k=2, \ldots, N_{s}-2, \\ 1-\frac{\Gamma\left(\left(N_{s}-2\right) m_{1}, \frac{m_{s}}{s^{\prime}} \gamma_{t h}\right)}{\Gamma\left(\left(N_{s}-2\right) m_{1}\right)}, & \text { if } k=N_{s}-1 .\end{cases}
$$

Note that the expression in Table I-(f) was obtained using [27, (3.191.1)] and [27, (3.383.1)].

2) Average Harvested Energy:

Substituting the PMF of $K$ derived in (60) in (11), we obtain the expression of the average harvested energy for the PDS scheme given by

$$
\bar{Q}^{(1)}=\zeta\left(\varepsilon_{s}+\varepsilon_{p}\right)\left[1+\sum_{j=1}^{N_{s}-2} \frac{\Gamma\left(j m_{1}, \frac{m_{1}}{\varepsilon_{s}} \gamma_{t h}\right)}{\Gamma\left(j m_{1}\right)}\right] .
$$

\section{3) Data Outage Probability:}

In order to derive the data outage probability, we need to derive the expressions of $\mathcal{I}_{1}\left(\xi_{s}, \gamma_{t h}\right), \mathcal{I}_{k}\left(\xi_{s}, \gamma_{t h}\right)$ and $\mathcal{I}_{N_{s}-1}\left(\xi_{s}, \gamma_{t h}\right)$. Let us start by $\mathcal{I}_{1}\left(\xi_{s}, \gamma_{t h}\right)$ defined in (76). We show that

$$
\begin{aligned}
\mathcal{I}_{1}\left(\xi_{s}, \gamma_{t h}\right)= & \frac{\Gamma\left(m_{1}, \frac{m_{1}}{\varepsilon_{s}} \gamma_{t h}\right)}{\Gamma\left(m_{1}\right)} \frac{\Gamma\left(m_{2}, \frac{m_{2}}{\varepsilon_{p}} \frac{\gamma_{t h}}{\xi_{s}}\right)}{\Gamma\left(m_{2}\right)} \\
& -\frac{\left(\frac{m_{2}}{\varepsilon_{p}}\right)^{m_{2}}}{\Gamma\left(m_{2}\right)} \sum_{j=0}^{m_{1}-1} \frac{\Gamma\left(m_{2}+j, \frac{\gamma_{t h}}{\xi_{s}}\left(\frac{m_{2}}{\varepsilon_{p}}+\frac{m_{1} \xi_{s}}{\varepsilon_{s}}\right)\right)}{\left(\frac{\varepsilon_{s}}{m_{1} \xi_{s}}\right)^{j}\left(\frac{m_{2}}{\varepsilon_{p}}+\frac{m_{1} \xi_{s}}{\varepsilon_{s}}\right)^{m_{2}+j} \Gamma(j+1)},
\end{aligned}
$$

which was obtained using the expression in Table I-(g).

Next, we compute $\mathcal{I}_{k}\left(\xi_{s}, \gamma_{t h}\right)$ as defined in (78):

$$
\begin{aligned}
& \mathcal{I}_{k}\left(\xi_{s}, \gamma_{t h}\right)=\frac{\Gamma\left(m_{2} k, \frac{m_{2}}{\varepsilon_{p}} \frac{\gamma_{t h}}{\xi_{s}}\right)}{\Gamma\left(m_{2} k\right)}\left(\frac{\Gamma\left(m_{1} k, \frac{m_{1}}{\varepsilon_{s}} \gamma_{t h}\right)}{\Gamma\left(m_{1} k\right)}-\frac{\Gamma\left(m_{1}(k-1), \frac{m_{1}}{\varepsilon_{s}} \gamma_{t h}\right)}{\Gamma\left(m_{1}(k-1)\right)}\right) \\
& -\frac{\left(\frac{m_{2}}{\varepsilon_{p}}\right)^{m_{2} k} e^{\frac{m_{1}}{\varepsilon_{s}} \gamma_{t h}} \Gamma\left(m_{2} k, \frac{\gamma_{t h}}{\xi_{s}}\left(\frac{m_{2}}{\varepsilon_{p}}+\frac{m_{1} \xi_{s}}{\varepsilon_{s}}\right)\right)}{\left(\frac{m_{2}}{\varepsilon_{p}}+\frac{m_{1} \xi_{s}}{\varepsilon_{s}}\right)^{m_{2} k}}\left(\frac{\Gamma\left(m_{1} k, \frac{m_{1}}{\varepsilon_{s}} \gamma_{t h}\right)}{\Gamma\left(m_{1} k\right)}-\frac{\Gamma\left(m_{1}, \frac{m_{1}}{\varepsilon_{s}} \gamma_{t h}\right)}{\Gamma\left(m_{1}\right)}\right) \\
& -\frac{\left(\frac{m_{2}}{\varepsilon_{p}}\right)^{m_{2} k}}{\Gamma\left(m_{2} k\right)} \sum_{j=0}^{m_{1}-1} \frac{\Gamma\left(m_{2} k+j, \frac{\gamma_{t h}}{\xi_{s}}\left(\frac{m_{2}}{\varepsilon_{p}}+\frac{m_{1} \xi_{s}}{\varepsilon_{s}}\right)\right)}{\left(\frac{\varepsilon_{s}}{m_{1} \xi_{s}}\right)^{j}\left(\frac{m_{2}}{\varepsilon_{p}}+\frac{m_{1} \xi_{s}}{\varepsilon_{s}}\right)^{m_{2} k+j} \Gamma(j+1)} \\
& +\frac{\Gamma\left(m_{1}(k-1), \frac{m_{1}}{\varepsilon_{s}} \gamma_{t h}\right)}{\Gamma\left(m_{1}(k-1)\right)} \frac{\left(\frac{m_{2}}{\varepsilon_{p}}\right)^{m_{2} k} e^{\frac{m_{1}}{\varepsilon_{s}} \gamma_{t h}}}{\Gamma\left(m_{2} k\right)} \sum_{j=0}^{m_{1}-1} \frac{1}{\left(\frac{\varepsilon_{s}}{m_{1} \xi_{s}}\right)^{j} \Gamma(j+1)} \\
& \times \sum_{n=0}^{j}\left(\begin{array}{l}
j \\
n
\end{array}\right)\left(-\frac{\gamma_{t h}}{\xi_{s}}\right)^{j-n} \frac{\Gamma\left(m_{2} k+n, \frac{\gamma_{t h}}{\xi_{s}}\left(\frac{m_{2}}{\varepsilon_{p}}+\frac{m_{1} \xi_{s}}{\varepsilon_{s}}\right)\right)}{\left(\frac{m_{2}}{\varepsilon_{p}}+\frac{m_{1} \xi_{s}}{\varepsilon_{s}}\right)^{m_{2} k+n}}
\end{aligned}
$$

which was obtained using the expressions in Table I-(g), Table I-(h), and Table I-(i).

Finally, we compute $I_{N_{s}-1}\left(\xi_{s}, \gamma_{t h}\right)$ as defined in (81):

$$
\mathcal{I}_{N_{s}-1}\left(\xi_{s}, \gamma_{t h}\right)=1
$$

$$
\begin{aligned}
& -\frac{\left(\frac{m_{2}}{\varepsilon_{p}}\right)^{m_{2}\left(N_{s}-1\right)}}{\Gamma\left(m_{2}\left(N_{s}-1\right)\right)} \sum_{j=0}^{m_{1}-1} \frac{\Gamma\left(m_{2}\left(N_{s}-1\right)+j, \frac{\gamma_{t h}}{\xi_{s}}\left(\frac{m_{2}}{\varepsilon_{p}}+\frac{m_{1} \xi_{s}}{\varepsilon_{s}}\right)\right)}{\left(\frac{\varepsilon_{s}}{m_{1} \xi_{s}}\right)^{j}\left(\frac{m_{2}}{\varepsilon_{p}}+\frac{m_{1} \xi_{s}}{\varepsilon_{s}}\right)^{m_{2}\left(N_{s}-1\right)+j} \Gamma(j+1)} \\
& -\frac{\left(\frac{m_{2}}{\varepsilon_{p}}\right)^{m_{2}\left(N_{s}-1\right)}}{\Gamma\left(m_{2}\left(N_{s}-1\right)\right)} \sum_{j=0}^{m_{1}\left(N_{s}-1\right)-1} \frac{\gamma\left(m_{2}\left(N_{s}-1\right)+j, \frac{\gamma_{t h}}{\xi_{s}}\left(\frac{m_{2}}{\varepsilon_{p}}+\frac{m_{1} \xi_{s}}{\varepsilon_{s}}\right)\right)}{\left(\frac{\varepsilon_{s}}{m_{1} \xi_{s}}\right)^{j}\left(\frac{m_{2}}{\varepsilon_{p}}+\frac{m_{1} \xi_{s}}{\varepsilon_{s}}\right)^{m_{2}\left(N_{s}-1\right)+j} \Gamma(j+1)} \\
& -\frac{\left(\frac{m_{2}}{\varepsilon_{p}}\right)^{m_{2}\left(N_{s}-1\right)} e^{\frac{m_{1}}{\varepsilon_{s}} \gamma_{t h}} \Gamma\left(m_{2}\left(N_{s}-1\right), \frac{\gamma_{t h}}{\xi_{s}}\left(\frac{m_{2}}{\varepsilon_{p}}+\frac{m_{1} \xi_{s}}{\varepsilon_{s}}\right)\right)}{\left(\frac{m_{2}}{\varepsilon_{p}}+\frac{m_{1} \xi_{s}}{\varepsilon_{s}}\right)^{m_{2}\left(N_{s}-1\right)}} \\
& \times\left(\frac{\Gamma\left(m_{1}\left(N_{s}-1\right), \frac{m_{1}}{\varepsilon_{s}} \gamma_{t h}\right)}{\Gamma\left(m_{1}\left(N_{s}-1\right)\right)}-\frac{\Gamma\left(m_{1}, \frac{m_{1}}{\varepsilon_{s}} \gamma_{t h}\right)}{\Gamma\left(m_{1}\right)}\right) \\
& -\frac{\Gamma\left(m_{1}\left(N_{s}-2\right), \frac{m_{1}}{\varepsilon_{s}} \gamma_{t h}\right)}{\Gamma\left(m_{1}\left(N_{s}-2\right)\right)}\left[\frac{\Gamma\left(m_{2}\left(N_{s}-1\right), \frac{m_{2}}{\varepsilon_{p}} \frac{\gamma_{t h}}{\xi_{s}}\right)}{\Gamma\left(m_{2}\left(N_{s}-1\right)\right)}\right.
\end{aligned}
$$


- If $\varepsilon_{s}=\varepsilon_{p}$,

$$
\begin{aligned}
& \mathcal{P} \mathcal{O}^{(3)}\left(\gamma_{q}\right)=\sum_{k=1}^{N_{s}-2} \frac{\left(\frac{\gamma_{t h}}{\varepsilon_{s}}\right)^{k-1} e^{-\frac{\gamma_{t h}}{\varepsilon_{s}}}}{\Gamma\left(N_{s}-k\right) \varepsilon_{s}^{N_{s}-k} \Gamma(k)}\left[\varepsilon_{s}^{N_{s}-k}\left(\frac{\Gamma\left(N_{s}-k\right)}{\Gamma\left(2\left(N_{s}-k\right)\right)} \gamma\left(2\left(N_{s}-k\right), \frac{\gamma_{q}}{\zeta \varepsilon_{s}}\right)-\gamma\left(N_{s}-k, \frac{\gamma_{q}}{\zeta \varepsilon_{s}}\right)+\gamma\left(N_{s}-k, \frac{\frac{\gamma_{q}}{\zeta}-\gamma_{t h}}{\varepsilon_{s}}\right)\right)\right. \\
& \left.+\left(\frac{\gamma_{q}}{\zeta}-\gamma_{t h}\right)^{N_{s}-k-1} e^{-\frac{\gamma_{q}}{\zeta \varepsilon_{s}}} \sum_{j=0}^{N_{s}-k-1} \frac{\gamma_{t h}^{j+1}{ }_{2} F_{1}\left(1,-N_{s}+k+1 ; j+2 ;-\frac{\gamma_{t h}}{\zeta \frac{\gamma_{q}}{\zeta}-\gamma_{t h}}\right)}{\Gamma(j+2) \varepsilon_{s}^{j}}\right]-\sum_{k=1}^{N_{s}-2} \frac{\left(\frac{\gamma_{t h}}{\varepsilon_{s}}\right)^{k-1} e^{-\frac{\gamma_{t h}}{\varepsilon_{s}}} \gamma\left(N_{s}-k, \frac{\frac{\gamma_{q}}{\zeta}-\gamma_{t h}}{\varepsilon_{p}}\right) \gamma\left(N_{s}-k, \frac{\gamma_{t h}}{\varepsilon_{s}}\right)}{\Gamma\left(N_{s}-k\right)^{2} \Gamma(k)} \\
& +\frac{\gamma\left(N_{s}-2, \frac{\gamma_{t h}}{\varepsilon_{s}}\right) e^{-\frac{\gamma_{q}}{\varepsilon \varepsilon_{p}}}\left(\frac{\gamma_{q}}{\zeta}-\gamma_{t h}\right)}{\Gamma\left(N_{s}-2\right) \varepsilon_{s}}-\frac{e^{-\frac{\frac{\gamma_{q}}{\zeta}-\gamma_{t h}}{\varepsilon_{p}}} e^{-\frac{\gamma_{t h}}{\varepsilon_{s}}} \gamma\left(N_{s}-2, \frac{\gamma_{t h}}{\varepsilon_{s}}\right)}{\Gamma\left(N_{s}-2\right)}+\sum_{k=1}^{N_{s}-1} \mathcal{J}_{k}\left(\gamma_{q}, \gamma_{t h}\right) \frac{\gamma\left(k, \frac{\gamma_{t h}}{\varepsilon_{s}}\right)}{\Gamma(k)} \text {. }
\end{aligned}
$$

- If $\varepsilon_{s} \neq \varepsilon_{p}$,

$$
\begin{aligned}
& \mathcal{P} O^{(3)}\left(\gamma_{q}\right)=\sum_{k=1}^{N_{s}-2} \frac{\left(\frac{\gamma_{t h}}{\varepsilon_{s}}\right)^{k-1} e^{-\frac{\gamma_{t h}}{\varepsilon_{s}}}}{\Gamma\left(N_{s}-k\right) \varepsilon_{s}^{N_{s}-k} \Gamma(k)}\left[\varepsilon_{s}^{N_{s}-k} \gamma\left(N_{s}-k, \frac{\frac{\gamma_{q}}{\zeta}-\gamma_{t h}}{\varepsilon_{s}}\right)-e^{-\frac{\gamma_{q}}{\zeta \varepsilon_{p}}} \sum_{j=0}^{N_{s}-k-1} \sum_{l=0}^{j}\left(\begin{array}{l}
j \\
l
\end{array}\right) \frac{\left(\frac{\gamma_{q}}{\zeta}\right)^{j-l}(-1)^{l} \gamma\left(N_{s}-k+l,\left(\frac{\gamma_{q}}{\zeta}-\gamma_{t h}\right)\left(\frac{1}{\varepsilon_{s}}-\frac{1}{\varepsilon_{p}}\right)\right)}{\Gamma(j+1) \varepsilon_{p}^{j}\left(\frac{1}{\varepsilon_{s}}-\frac{1}{\varepsilon_{p}}\right)^{N_{s}-k+l}}\right] \\
& -\sum_{k=1}^{N_{s}-2} \frac{\left(\frac{\gamma_{t h}}{\varepsilon_{s}}\right)^{k-1} e^{-\frac{\gamma_{t h}}{\varepsilon_{s}}} \gamma\left(N_{s}-k, \frac{\frac{\gamma_{q}}{\zeta}-\gamma_{t h}}{\varepsilon_{p}}\right) \gamma\left(N_{s}-k, \frac{\gamma_{t h}}{\varepsilon_{s}}\right)}{\Gamma\left(N_{s}-k\right)^{2} \Gamma(k)}+\frac{\gamma\left(N_{s}-2, \frac{\gamma_{t h}}{\varepsilon_{s}}\right) e^{-\frac{\gamma_{q}}{\varepsilon_{p}}}}{\Gamma\left(N_{s}-2\right) \varepsilon_{s}} \frac{1-e^{-\left(\frac{\gamma_{q}}{\zeta}-\gamma_{t h}\right)\left(\frac{1}{\varepsilon_{s}}-\frac{1}{\varepsilon_{p}}\right)}}{\left(\frac{1}{\varepsilon_{s}}-\frac{1}{\varepsilon_{p}}\right)}-\frac{e^{-\frac{\gamma_{q}}{\zeta}-\gamma_{t h}} \frac{\gamma_{p}}{\varepsilon_{p}} e^{-\frac{\gamma_{t h}}{\varepsilon_{s}}} \gamma\left(N_{s}-2, \frac{\gamma_{t h}}{\varepsilon_{s}}\right)}{\Gamma\left(N_{s}-2\right)} \\
& +\sum_{k=1}^{N_{s}-1} \mathcal{J}_{k}\left(\gamma_{q}, \gamma_{t h}\right) \frac{\gamma\left(k, \frac{\gamma_{t h}}{\varepsilon_{s}}\right)}{\Gamma(k)} .
\end{aligned}
$$

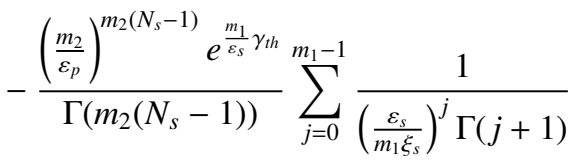

$$
\begin{aligned}
& \left.\times \sum_{n=0}^{j}\left(\begin{array}{l}
j \\
n
\end{array}\right)\left(-\frac{\gamma_{t h}}{\xi_{s}}\right)^{j-n} \frac{\Gamma\left(m_{2}\left(N_{s}-1\right)+n, \frac{\gamma_{t h}}{\xi_{s}}\left(\frac{m_{2}}{\varepsilon_{p}}+\frac{m_{1} \xi_{s}}{\varepsilon_{s}}\right)\right)}{\left(\frac{m_{2}}{\varepsilon_{p}}+\frac{m_{1} \xi_{s}}{\varepsilon_{s}}\right)^{m_{2}\left(N_{s}-1\right)+n}}\right],
\end{aligned}
$$

which was obtained using the expressions in Table I-(e), Table I-(f), Table I-(g), and Table I-(h).

\section{B. PES Scheme}

\section{1) $P M F$ of $K$ :}

The PMF of $K$ for the PES scheme can be immediately obtained given the fact $P_{K}^{(2)}(k)=P_{K}^{(1)}\left(N_{s}-k\right)$. Hence, we can have

$$
\begin{aligned}
& P_{K}^{(2)}(k) \\
& = \begin{cases}1-\frac{\Gamma\left(\left(N_{s}-2\right) m_{1}, \frac{m_{1}}{\varepsilon_{s}} \gamma_{t h}\right)}{\Gamma\left(\left(N_{s}-2\right) m_{1}\right)}, & \text { if } k=1, \\
\frac{\Gamma\left(\left(N_{s}-k\right) m_{1}, \frac{m_{1}}{\varepsilon_{s}} \gamma_{t h}\right)}{\Gamma\left(\left(N_{s}-k\right) m_{1}\right)}-\frac{\Gamma\left(\left(N_{s}-k-1\right) m_{1}, \frac{m_{1}}{\varepsilon_{s}} \gamma_{t h}\right)}{\Gamma\left(\left(N_{s}-k-1\right) m_{1}\right)}, & \text { if } k=2, \ldots, N_{s}-2, \\
\frac{\Gamma\left(m_{1}, \frac{m_{1}}{\varepsilon_{s}} \gamma_{t h}\right)}{\Gamma\left(m_{1}\right)}, & \text { if } k=N_{s}-1 .\end{cases}
\end{aligned}
$$

\section{2) Average Harvested Energy:}

Using the fact that $\bar{Q}^{(2)}=\zeta\left(\varepsilon_{s}+\varepsilon_{p}\right) N_{s}-\bar{Q}^{(1)}$, we obtain

$$
\bar{Q}^{(2)}=\zeta\left(\varepsilon_{s}+\varepsilon_{p}\right)\left[N_{s}-1-\sum_{j=1}^{N_{s}-2} \frac{\Gamma\left(j m_{1}, \frac{m_{1}}{\varepsilon_{s}} \gamma_{t h}\right)}{\Gamma\left(j m_{1}\right)}\right] .
$$

3) Data Outage Probability:

Using [27, (6.455.1)], we can show that the conditional data outage probability is given by

$$
P_{\text {out }}^{(2)}\left(\xi_{s} \mid k=1, \ldots, N_{s}-1\right)
$$

$$
\begin{aligned}
& =1-\frac{\left(\frac{m_{2}}{\varepsilon_{p}}\right)^{k m_{2}}\left(\frac{m_{1} \xi_{s}}{\varepsilon_{s}}\right)^{k m_{1}} \Gamma\left(k\left(m_{1}+m_{2}\right)\right)}{\Gamma\left(k m_{2}+1\right) \Gamma\left(k m_{1}\right)\left(\frac{m_{2}}{\varepsilon_{p}}+\xi_{s} \frac{m_{1}}{\varepsilon_{s}}\right)^{k\left(m_{1}+m_{2}\right)}} \\
& \times{ }_{2} F_{1}\left(1, k\left(m_{1}+m_{2}\right) ; k m_{2}+1 ; \frac{m_{2} \varepsilon_{s}}{m_{2} \varepsilon_{s}+\xi_{s} m_{1} \varepsilon_{p}}\right) .
\end{aligned}
$$

Subsequently, the data outage probability for the PES scheme is given by (24).

\section{Special Case: Scalability at ST and PT}

Here, we consider the case where ST and PT are equipped with multiple antennas, $N_{1}$ and $N_{p}$, respectively. All the STSR and PT-SR channels are independent identically distributed (i.i.d). All the channels are combined at SR and they can be seen as Nakagami fading channels with parameters $m_{1}=N_{1}$, $m_{2}=N_{p}, \bar{\lambda}_{s s}=N_{1} \varepsilon_{1}$, and $\bar{\lambda}_{p s}=N_{p} \varepsilon_{2}$, where varepsilon vand $_{1}$ and $\varepsilon_{2}$ are the channel gain per branch between ST and SR and the channel gain per branch between PT and SR, respectively. varepsilon $_{1}$ and $\varepsilon_{2}$ are the channel variances if the Rayleigh fading example is considered. Subsequently, we can obtain the energy and data performance analysis of the PDS and PES schemes.

\section{Simulation Results}

In this section, we present some selected simulations to show the accuracy of the obtained analytical expressions compared to the Monte Carlo (MC) simulation and to compare performance of the two PDS and PES selection schemes. The number of simulations is $N_{\text {sim }}=10^{4}$. The conversion efficiency of the EH circuits at SR is $\zeta=60 \%$. The transmission rate at $\mathrm{ST}$ is chosen equal to $2 \mathrm{bps} / \mathrm{Hz}$. 


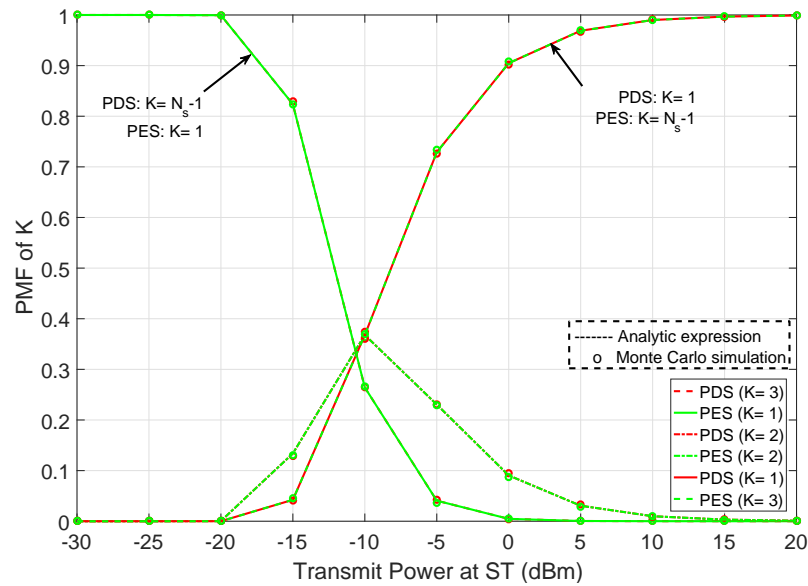

Figure 2: The probability mass function of $K$ versus the transmit power $P_{s}$ at ST in dBm, with $N_{s}=4, P_{p}=10 \mathrm{dBm}$, $\lambda_{s s}=\lambda_{p s}=30 \mathrm{dBm}$, and $\gamma_{t h}=-10 \mathrm{dBm}, \gamma_{q}=10 \mathrm{dBm}$ for the two PDS and PES selection schemes.

\section{A. Rayleigh Fading Channels}

In this part, the channels follow the Rayleigh distribution.

In Fig. 2, we plotted the PMF of $K$ versus the transmit power $P_{s}$ at $\mathrm{ST}$ in $\mathrm{dBm}$, respectively, for $N_{s}=4, P_{p}=10$ $\mathrm{dBm}, \lambda_{s s}=\lambda_{p s}=30 \mathrm{dBm}$, and $\gamma_{t h}=-10 \mathrm{dBm}$ for the two PDS and PES selection schemes. We can see that for the low power regime, $K$ goes to $N_{s}-1$ for the PDS scheme and goes to 1 for the PES scheme. However, for the high power regime, $K$ goes to 1 for the PDS scheme and goes to $N_{s}-1$ for the PES scheme.

1) Threshold $\gamma_{t h}$ : In Figs. 3a, 3b, 3c, we plotted the data outage probability, the average harvested energy and the power outage versus the threshold $\gamma_{t h}$, respectively, for $N_{s}=10$, $P_{s}=P_{p}=10 \mathrm{dBm}, \lambda_{s s}=\lambda_{p s}=30 \mathrm{dBm}$, and $\gamma_{q}=10$ $\mathrm{dBm}$. We have plotted different ways to select $K$ to show the relevance of our proposed PDS and PES schemes: the fixed/random $K$, the SIR based selection scheme which selects $K$ that maximizes the SIR, the TS scheme which separates the $\mathrm{EH}$ and ID tasks at SR over time and the EH time ratio is $\alpha=0.5$, and the joint PDS and PES scheme applied together where $K=\min \left(K^{(1)}, K^{(2)}\right)$ with $K^{(1)}$ and $K^{(2)}$ are the ones chosen by the PDS scheme and the PES scheme, respectively. In terms of the data outage probability, we can see that one of our proposed schemes have the closest performance to the SIR based selection scheme and outperform all the other selection methods. For low values of the threshold, the PDS scheme is the closest scheme to the SIR-based scheme. For high values of the threshold, the PES scheme is the closest one to the SIR based scheme. Also, in terms of the energy metrics, either the PDS scheme or the PES scheme outperform all the other methods. Moreover, the joint PDS ans PES scheme unifies all the advantages of the two PDS and PES schemes and it has the best data and energy performance compared to the other selection schemes, except the SIR based scheme which outperforms all schemes in terms of data outage. Also, we can see that as $\gamma_{t h}$ increases, the data outage probability of the PDS scheme improves, while it increases for the PES scheme.

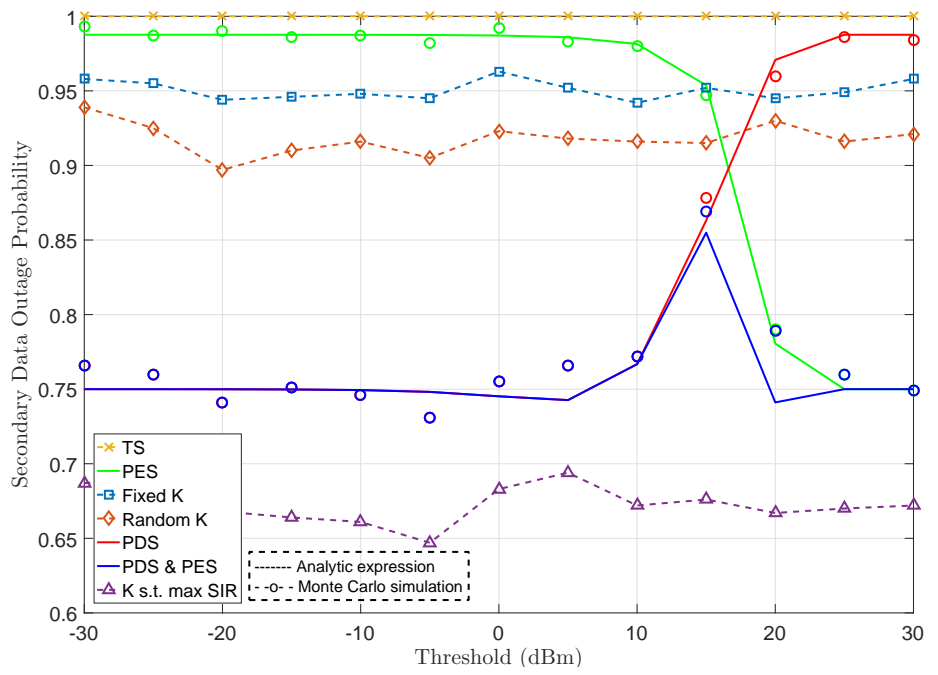

(a) The data outage probability $P_{\text {out }}$.

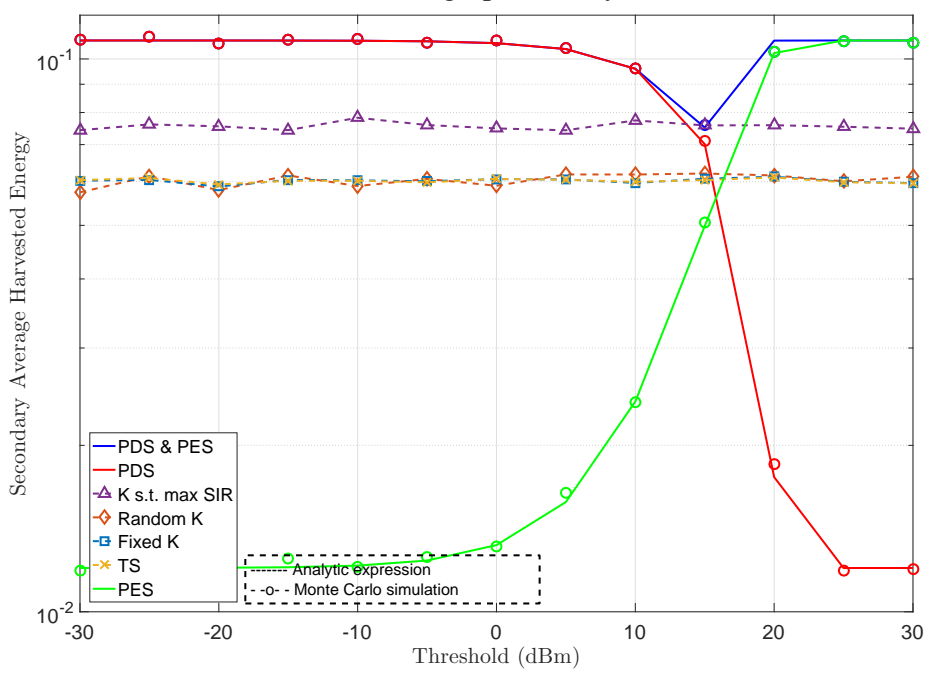

(b) The average harvested energy $\bar{Q}$.

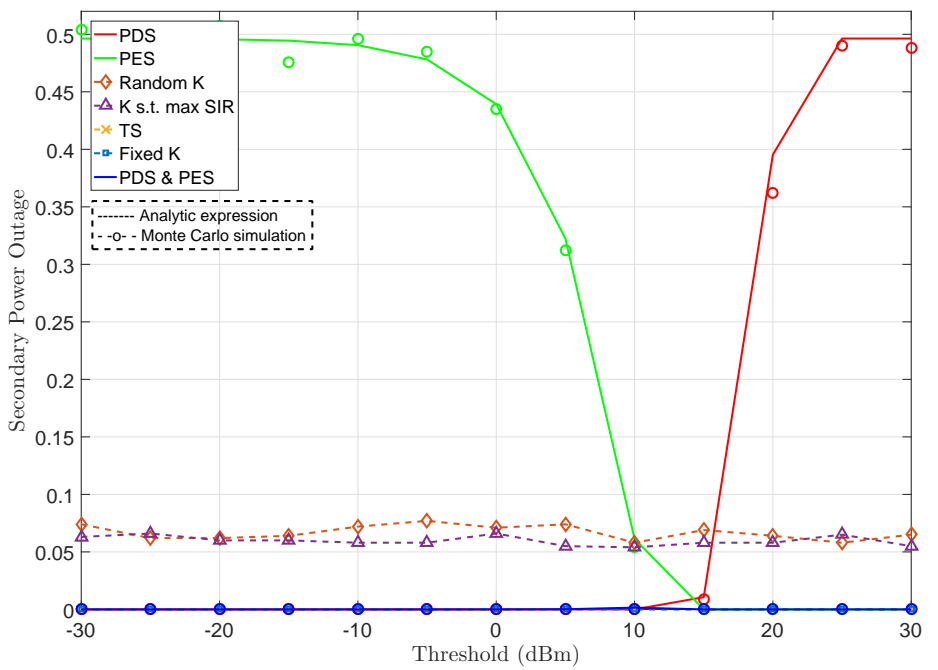

(c) The power outage probability $\mathcal{P} O$.

Figure 3: (a) The data outage probability $P_{\text {out }}$, (b) the average harvested energy $\bar{Q}$, and (c) the power outage probability $\mathcal{P O}$ versus the threshold $\gamma_{t h}$ in $\mathrm{dBm}$, with $N_{s}=10, P_{s}=P_{p}=10$ $\mathrm{dBm}, \lambda_{s s}=\lambda_{p s}=30 \mathrm{dBm}$, and $\gamma_{q}=10 \mathrm{dBm}$, for the two PDS and PES selection schemes. 


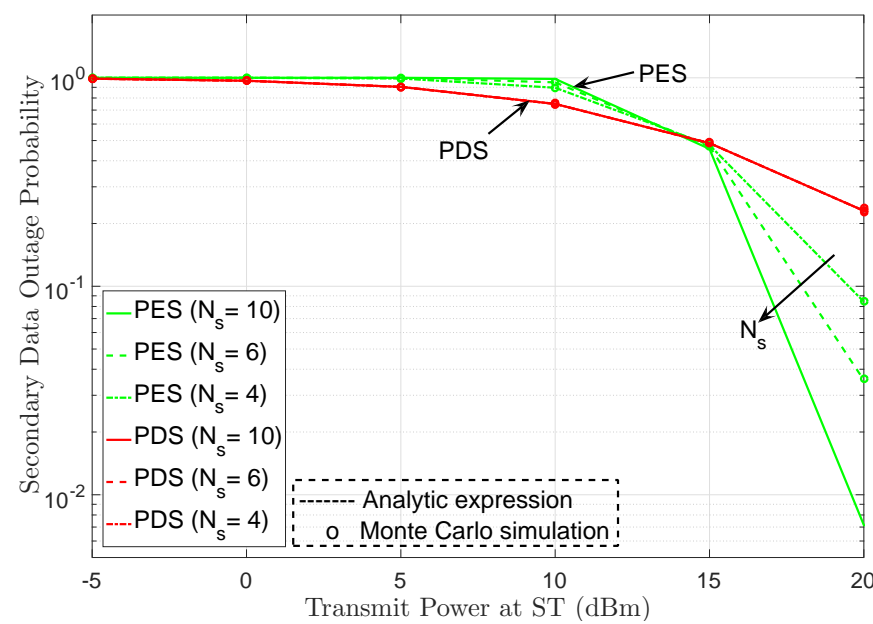

(a) The data outage probability $P_{\text {out }}$ with $\gamma_{t h}=-10 \mathrm{dBm}$.

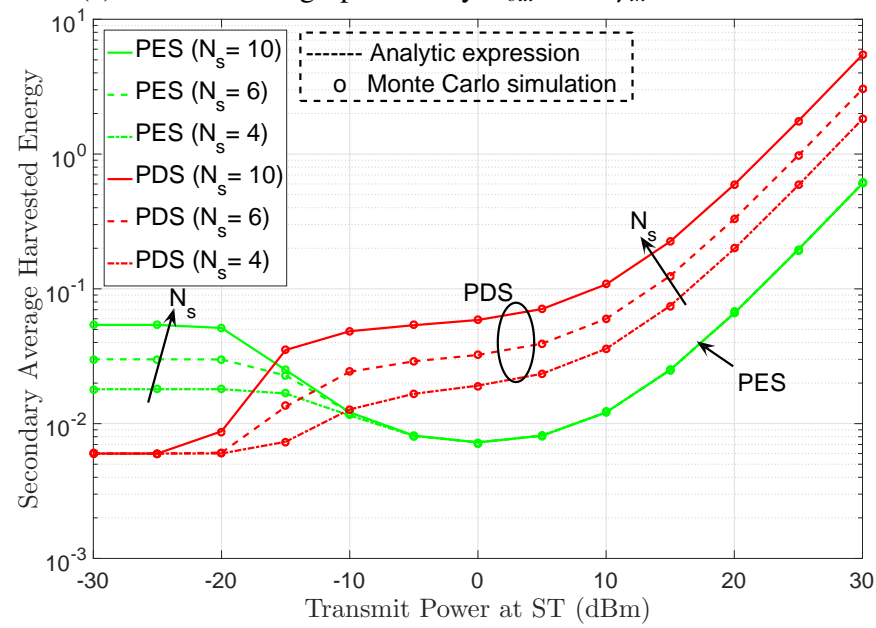

(b) The average harvested energy $\bar{Q}$ with $\gamma_{t h}=-10 \mathrm{dBm}$.

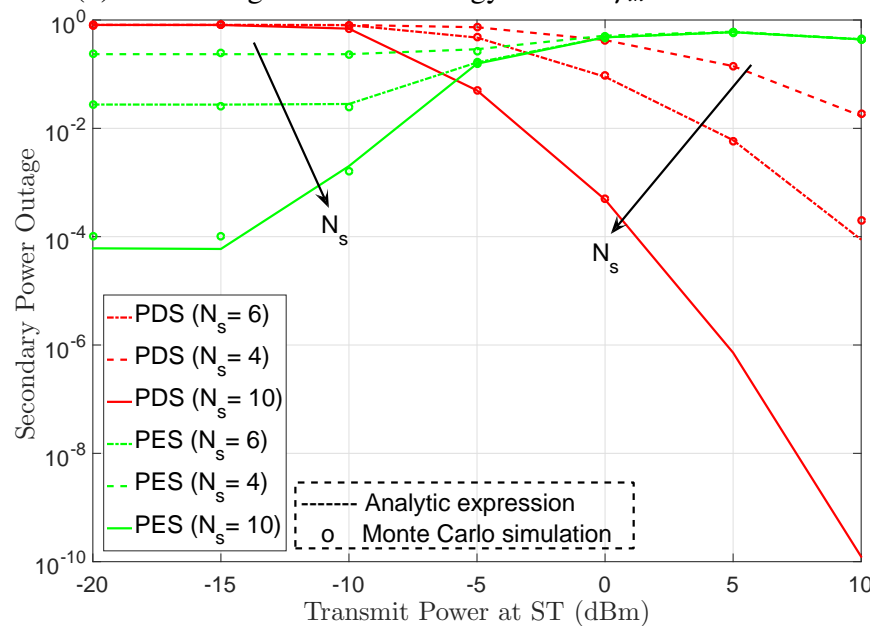

(c) The power outage probability $\mathcal{P O}$ with $\gamma_{q}=10 \mathrm{dBm}$ and $\gamma_{t h}=0$ $\mathrm{dBm}$.

Figure 4: (a) The data outage probability $P_{\text {out }}$, (b) the average harvested energy $\bar{Q}$, and (c) the power outage probability $\mathcal{P O}$ versus the transmit power $P_{s}$ at $\mathrm{ST}$ in $\mathrm{dBm}$, with $P_{p}=10$ $\mathrm{dBm}, \lambda_{s s}=\lambda_{p s}=30 \mathrm{dBm}, \gamma_{q}=10 \mathrm{dBm}$, and different values of $N_{s}$ for the two PDS and PES selection schemes.

On the other hand, the average harvested energy decreases for the PDS scheme and increases for the PES scheme, as $\gamma_{t h}$ increases. Also, the power outage probability increases for the PDS scheme and decreases for the PES scheme, as $\gamma_{t h}$ increases. This observation can be explained by the fact that as the threshold $\gamma_{t h}$ increases, more antennas are allocated to decode information for the PDS scheme while less antennas are allocated to harvest energy. However, for the PES scheme, as $\gamma_{t h}$ increases, more antennas are used to harvest energy and less antennas are used to decode information. Subsequently, as $\gamma_{t h}$ increases, the data metric improves for the PDS scheme and worsens for the PES scheme, while the energy metrics improve for the PES scheme and worsen for the PDS scheme.

2) Number of Antennas at SR: In Figs. 4a, 4b and 4c, we plotted the data outage probability, the average harvested energy and the power outage versus the transmit power $P_{s}$ at $\mathrm{ST}$ in $\mathrm{dBm}$, respectively, for $P_{p}=10 \mathrm{dBm}, \lambda_{s s}=\lambda_{p s}=30$ $\mathrm{dBm}$, and different values of $N_{s}$ for the two PDS and PES selection schemes. In Figs. $4 \mathrm{a}$, and $4 \mathrm{~b}$, we have $\gamma_{t h}=-10$ $\mathrm{dBm}$, while, in Fig. 4c, we have $\gamma_{t h}=0 \mathrm{dBm}$, and $\gamma_{q}=10$ $\mathrm{dBm}$. On the one hand, from a data outage probability stand point, we can see that the PDS scheme outperforms the PES scheme for a transmit power constraint $P_{s} \leq 15 \mathrm{dBm}$, while for $P_{s} \geq 15 d B$, the PES scheme outperforms the PDS scheme. On the other hand, in terms of the average harvested energy, the PES scheme outperforms the PDS scheme for $P_{s}<-10$ $\mathrm{dBm}$, while the PDS scheme outperforms the PES scheme for $P_{s} \geq-5 \mathrm{dBm}$. Also, in terms of the power outage probability, the PES scheme outperforms the PDS scheme for $P_{s}<-5$ $\mathrm{dBm}$, while the PDS scheme outperforms the PES scheme for $P_{s} \geq-10 \mathrm{dBm}$. This observation can be explained by the fact that, when the ratio $\frac{\gamma_{t h}}{\varepsilon_{s}}$ becomes very large (low power regime), the number of selected antennas $K$ of the PDS scheme converges to $N_{s}-1$, while it goes to 1 for the PES scheme. Hence, for the PDS scheme, more antennas are used to decode information and less are used to harvest energy. However, for the PES scheme, less antennas are used to decode information and more are used to harvest energy. Subsequently, the data outage probability of the PDS scheme is better than the one of the PES scheme, while the average harvested energy and the power outage probability of the PES scheme is better than the one the PDS scheme. However, when the ratio $\frac{\gamma_{t h}}{\varepsilon_{s}}$ becomes small (high power regime), the number of selected antennas $K$ for the PDS scheme converges to 1, while it goes to $N_{s}-1$ for the PES scheme. For the PDS scheme, only one antenna is used to decode the information and all the others are used to harvest energy. However, for the PES scheme, only one antenna is used to harvest energy and all the others are used to decode the information. Subsequently, the data outage probability of the PES scheme is better than the one of the PDS scheme, while the average harvested energy and the power outage probability of the PDS scheme is better than the one the PES scheme. At this point, we can see that there is a data-energy trade off when we use the PDS scheme or the PES scheme.

In addition, we can see that the data outage probability of the PDS scheme is almost the same, as we increase the number of receiving antennas at ST. For the high power regime, this behavior is expected from (36). 


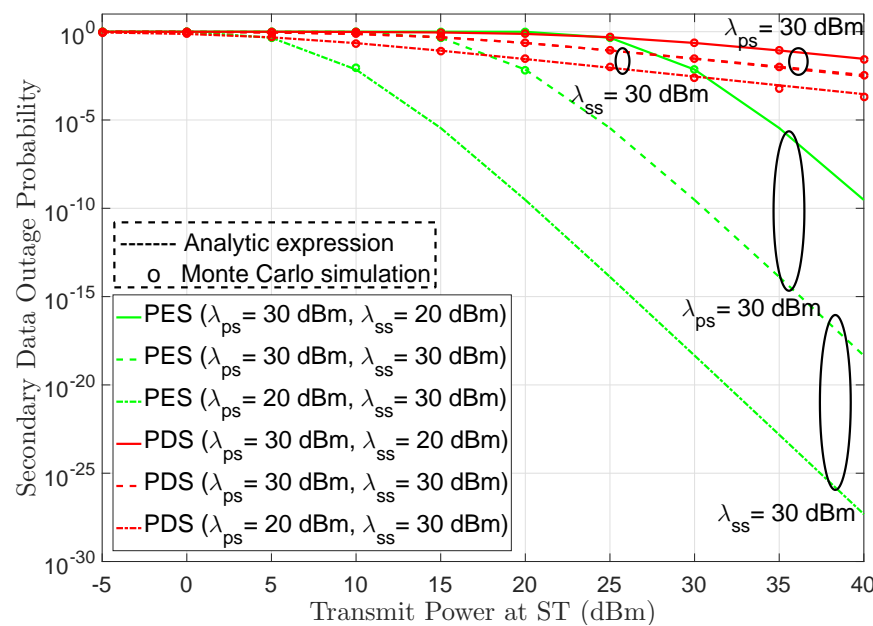

(a) The data outage probability $P_{\text {out }}$.

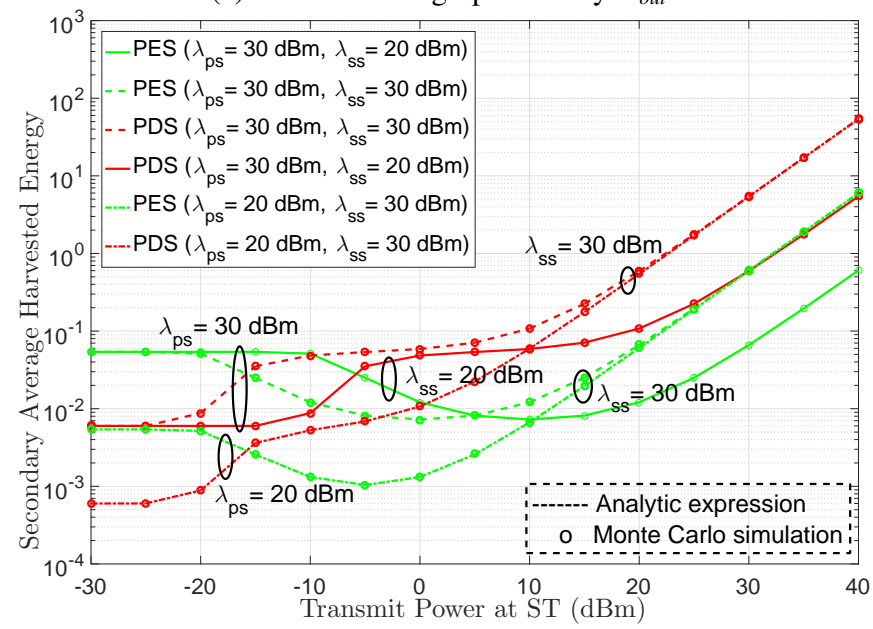

(b) The average harvested energy $\bar{Q}$.

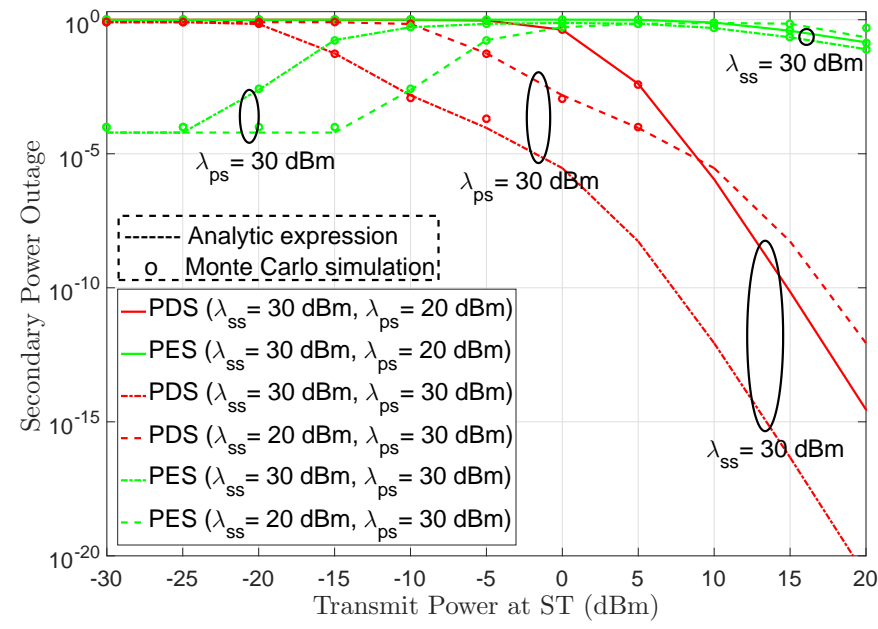

(c) The power outage probability $\mathcal{P O}$ with $\gamma_{q}=10 \mathrm{dBm}$.

Figure 5: (a) The data outage probability $P_{\text {out }}$ and (b) the average harvested energy $\bar{Q}$, and (c) the power outage probability $\mathcal{P O}$, versus the transmit power $P_{s}$ at ST in $\mathrm{dBm}$, with $N_{s}=10$, $P_{p}=10 \mathrm{dBm}, \gamma_{t h}=-10 \mathrm{dBm}, \gamma_{q}=10 \mathrm{dBm}$, and different values of $\lambda_{s s}$ and $\lambda_{p s}$ for the two PDS and PES selection schemes.

For the low power regime, this behavior is due to the fact that the ratio $\frac{\gamma_{t h}}{x \varepsilon_{p}}$ is of the order of $-50 \mathrm{dBm}$, so (39) converges to 1 . However, the data outage probability of the PES scheme increases for $P_{s} \leq 15 \mathrm{dBm}$, while it decreases for $P_{s}>15$ $\mathrm{dBm}$, as $N_{s}$ increases. On the other hand, as $N_{s}$ increases, the average harvested energy of the PDS scheme increases for $P_{s} \geq-25 \mathrm{dBm}$ and is almost constant otherwise, while the average harvested energy of the PES scheme increases for $P_{s} \leq-10 \mathrm{dBm}$ and is almost constant otherwise. Moreover, as $N_{s}$ increases, the power outage probability of the PDS scheme decreases for $P_{s} \geq-10 \mathrm{dBm}$ and is almost constant otherwise, while the power outage probability of the PES scheme decreases for $P_{s} \leq 0 \mathrm{dBm}$ and is almost constant otherwise. This constant behavior of the average harvested energy and the power outage probability with respect to $N_{s}$ is due to the fact that $K$ is equal to 1 when the PDS scheme is in the high power regime and when the PES scheme is in the low power regime.

3) Channel Variances $\lambda_{s s}$ and $\lambda_{p s}$ : In Figs. 5a, 5b, and 5c, we plotted the data outage probability, the average harvested energy the power outage probability versus the transmit power $P_{s}$ at $\mathrm{ST}$ in $\mathrm{dBm}$, respectively, for $N_{s}=10, P_{p}=10 \mathrm{dBm}$, $\gamma_{t h}=-10 \mathrm{dBm}, \gamma_{q}=10 \mathrm{dBm}$, and different values of $\lambda_{s s}$ and $\lambda_{p s}$ for the two PDS and PES selection schemes. We can see that the data outage probability of the PDS scheme and the PES scheme improves with $\lambda_{s s}$ while it worsens with $\lambda_{p s}$, which is an expected result since we know that the interference harms the data transmission. On the other hand, the average harvested energy and the power outage probability of the PDS scheme and the PES scheme improve with $\lambda_{p s}$, if we compare the cases when $\lambda_{p s}=20 \mathrm{dBm}$ and $\lambda_{p s}=30 \mathrm{dBm}$, while $\lambda_{s s}=30 \mathrm{dBm}$. But, when $\lambda_{s s}$ increases, the average harvested energy and the power outage probability of the PDS scheme improve. However, for the PES scheme, the average harvested energy and the power outage probability improve for $P_{s}>5$ $\mathrm{dBm}$ and worsen otherwise. Moreover, we can see that the average harvested energy of both PES and PDS schemes is highly affected by $\lambda_{p s}$ in the low power regime while it is highly affected by $\lambda_{s s}$ in the high power regime. Also, the power outage probability is highly affected by $\lambda_{p s}$ in the low power regime.

\section{B. Nakagami Fading Channels}

In this part, we consider the case of Nakagami fading channels.

In Fig. 6, we have plotted the data outage probability $P_{\text {out }}$ and the average harvested energy $\bar{Q}$ versus the transmit power $P_{s}$ at $\mathrm{ST}$ in $\mathrm{dBm}$, with $N_{s}=10, P_{p}=10 \mathrm{dBm}, \gamma_{t h}=0$ $\mathrm{dBm}, \gamma_{q}=10 \mathrm{dBm}, \bar{\lambda}_{s s}=\bar{\lambda}_{s s}=30 \mathrm{dBm}$, and different values of $m_{1}$ and $m_{2}$ for the two PDS and PES selection schemes. The ST-SR channels and the PT-SR channels follow the Nakagami fading channels. We can see first that increasing $m_{1}$ and $m_{2}$ worsens the data outage probability for a transmit power at ST less than $15 \mathrm{dBm}$. For high power regime, the data outage probability improves as $m_{1}$ and $m_{2}$ increase. Recall that when $m_{1}=m_{2}=1$, this case is equivalent to Rayleigh fading channels. In terms of the average harvested energy, the advantage due to increasing $m_{1}$ and $m_{2}$ is slightly observable. 


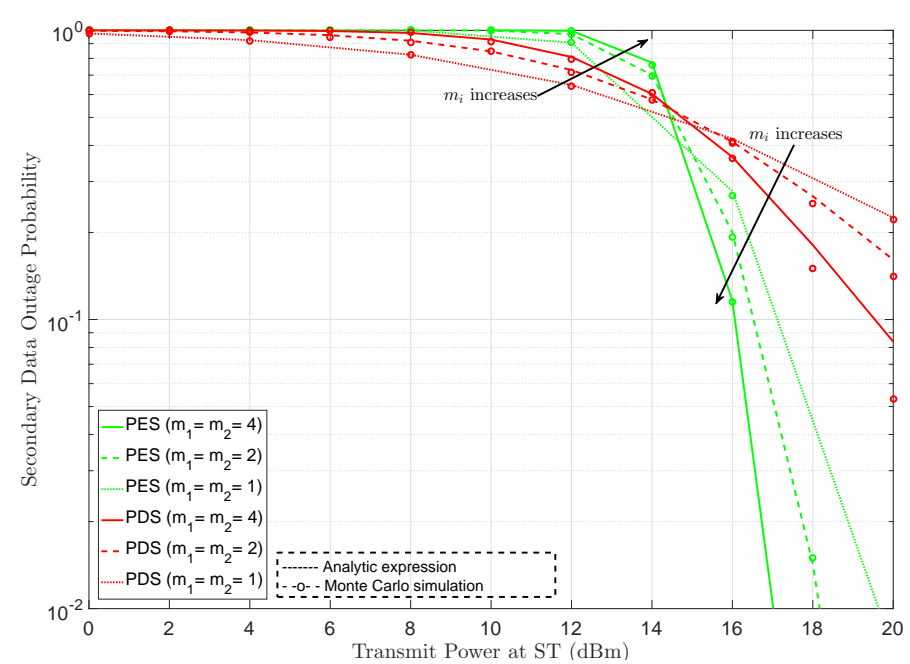

(a) The data outage probability $P_{\text {out }}$.

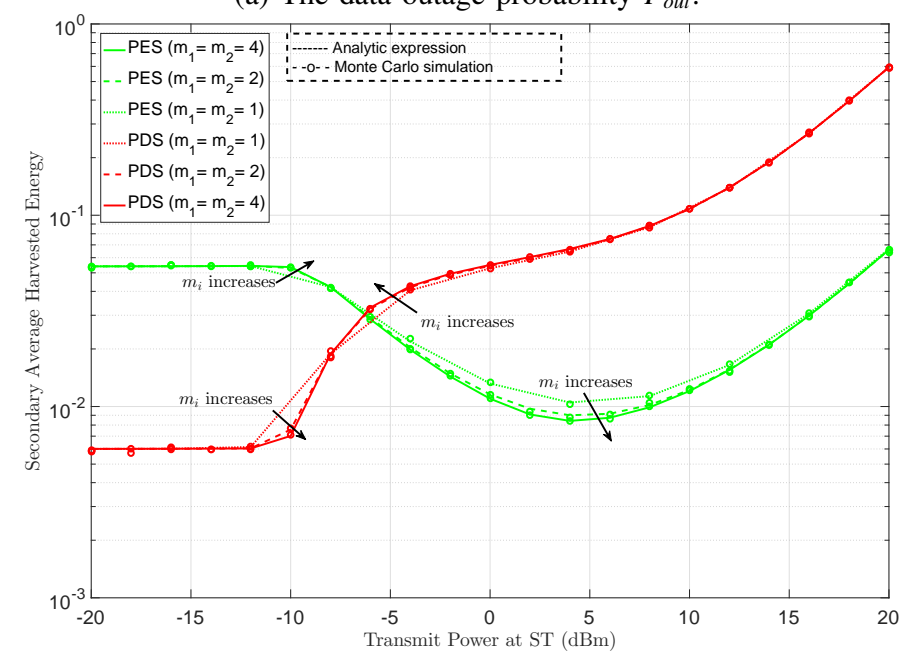

(b) The average harvested energy $\bar{Q}$.

Figure 6: (a) The data outage probability $P_{\text {out }}$ and (b) the average harvested energy $\bar{Q}$ versus the transmit power $P_{s}$ at ST in $\mathrm{dBm}$, with $N_{s}=10, P_{p}=10 \mathrm{dBm}, \gamma_{t h}=0 \mathrm{dBm}$, $\gamma_{q}=10 \mathrm{dBm}, \bar{\lambda}_{s s}=\bar{\lambda}_{p s}=30 \mathrm{dBm}$, and different values of $m_{1}$ and $m_{2}$ for the two PDS and PES selection schemes.

For low power regime, increasing $m_{1}$ and $m_{2}$ improves the average harvested energy of the PES scheme, while it worsens the average harvested energy of the PDS scheme. For high power regime, increasing $m_{1}$ and $m_{2}$ improves the average harvested energy of the PDS scheme, while it worsens the average harvested energy of the PES scheme.

In Fig. 7, we have plotted the data outage probability $P_{\text {out }}$ and the average harvested energy $\bar{Q}$ versus the threshold $\gamma_{t h}$ in $\mathrm{dBm}$, with $N_{s}=10, P_{s}=P_{p}=10 \mathrm{dBm}, \gamma_{q}=10$ $\mathrm{dBm}, \bar{\lambda}_{s s}=\bar{\lambda}_{p s}=30 \mathrm{dBm}$, and different values of $N_{1}$ and $N_{p}$ for the two PDS and PES selection schemes. ST and PT are equipped with $N_{1}$ and $N_{p}$ antennas, respectively. The ST-SR channels and the PT-SR channels are i.i.d. following the Rayleigh fading channels with channel gain per branch equal to $\bar{\lambda}_{s s}$ and $\bar{\lambda}_{p s}$, respectively. First, having more antennas at ST and PT worsens the data outage probability for the

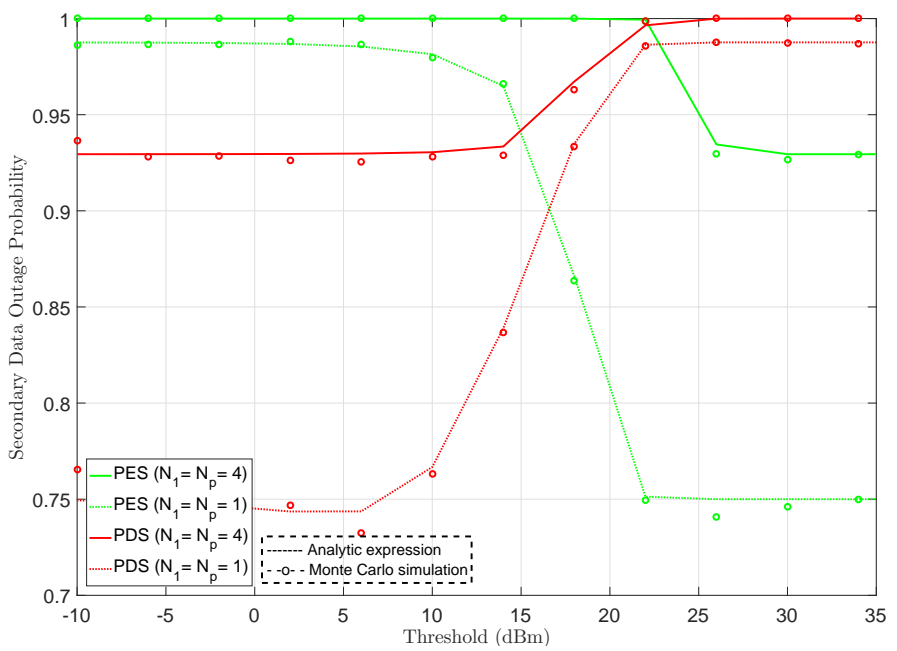

(a) The data outage probability $P_{\text {out }}$.

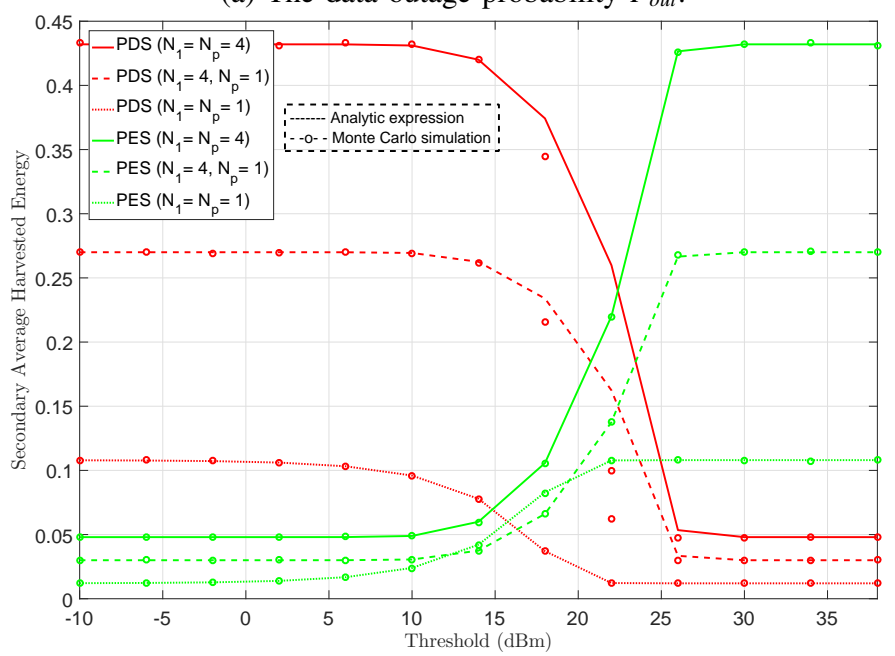

(b) The average harvested energy $\bar{Q}$.

Figure 7: (a) The data outage probability $P_{\text {out }}$ and (b) the average harvested energy $\bar{Q}$ versus the threshold $\gamma_{t h}$ in $\mathrm{dBm}$, with $N_{s}=10, P_{s}=P_{p}=10 \mathrm{dBm}, \gamma_{q}=10 \mathrm{dBm}, \bar{\lambda}_{s s}=\bar{\lambda}_{s s}=$ $30 \mathrm{dBm}$, and different values of $N_{1}$ and $N_{p}$ for the two PDS and PES selection schemes.

PDS scheme for low threshold and for the PES scheme for high threshold. This fact can be explained by the increasing interference due to PT transmission induced at SR. In terms of the average harvested energy, having more antennas at ST or PT improves the average harvested energy for the PDS scheme for low threshold and for the PES scheme for high threshold.

\section{Conclusion}

In this paper, we investigate the SWIPT for spectrum sharing in CR networks where the SR harvests the energy from the primary and secondary transmission using the antenna switching technique. For the AS technique, we propose a thresholding-based antenna selection strategy inspired from OT-MRC technique. We study two selection schemes: the first is the prioritizing data selection (PDS) scheme and the second is the prioritizing energy selection (PES) scheme. For both 
the PDS and PES schemes, we derive the expressions of the PMF of $K$, the average harvested energy, the power outage probability, and the data outage probability. We deduce the performance of the joint PDS ans PES scheme. We study the Rayleigh fading channel case and we derive some asymptotic results of the studied metrics. We study also the Nakagami fading channel case and we deduce the scalability case at ST and PT. Through the simulation results, we show the impact of different simulation parameters and we show that there is an energy-data trade off for both schemes. We compare the proposed selection schemes to some other methods to show the effectiveness of our methods.

\section{APPENDix A}

PDF AND CDF OF $Q^{(1)}(k)$ FOR $k=1, \ldots, N_{s}-1$

Recall, for $k=1, \ldots, N_{s}-1$, the harvested energy at SR is given by

$$
Q^{(1)}(k)=\zeta\left(\bar{\Gamma}_{s}(k)+\bar{\Gamma}_{p}(k)\right),
$$

where $\bar{\Gamma}_{s}(k)=P_{s} \sum_{k+1}^{N_{s}}\left|h_{s j}\right|^{2}, \bar{\Gamma}_{p}(k)=P_{p} \sum_{k+1}^{N_{s}}\left|h_{p j}\right|^{2}$ are the harvested energy from ST and PT, respectively.

The PDF and CDF of $\bar{\Gamma}_{s}(k)$ are denoted as $f_{\gamma_{s}}^{\left(N_{s}-k\right)}(\cdot)$, $F_{\gamma_{s}}^{\left(N_{s}-k\right)}(\cdot)$, respectively. In addition, the PDF and CDF of $\bar{\Gamma}_{p}(k)$ are denoted as $f_{\gamma_{p}}^{\left(N_{s}-k\right)}(\cdot), F_{\gamma_{p}}^{\left(N_{s}-k\right)}(\cdot)$, respectively.

We can write the PDF and CDF of $Q^{(1)}(k)$ as

$$
\begin{gathered}
f_{Q^{(1)}(k)}(q)=\frac{1}{\zeta} \int_{0}^{\frac{q}{\zeta}} f_{\gamma_{s}}^{\left(N_{s}-k\right)}(x) f_{\gamma_{p}}^{\left(N_{s}-k\right)}\left(\frac{q}{\zeta}-x\right) \mathrm{d} x, \\
F_{Q^{(1)}(k)}\left(\gamma_{q}\right)=\int_{0}^{\frac{\gamma_{q}}{\zeta}} f_{\gamma_{s}}^{\left(N_{s}-k\right)}(x) F_{\gamma_{p}}^{\left(N_{s}-k\right)}\left(\frac{\gamma_{q}}{\zeta}-x\right) \mathrm{d} x,
\end{gathered}
$$

respectively.

For the Rayleigh fading channels, the PDF of $Q^{(1)}(k)$ is written as

$$
\begin{aligned}
f_{Q^{(1)}(k)}(q) & =\frac{e^{-\frac{q}{\zeta \varepsilon_{p}}}}{\zeta \Gamma\left(N_{s}-k\right)^{2} \varepsilon_{s}^{N_{s}-k} \varepsilon_{p}^{N_{s}-k}} \int_{0}^{\frac{q}{\zeta}} x^{N_{s}-k-1}\left(\frac{q}{\zeta}-x\right)^{N_{s}-k-1} \\
& \times e^{-x\left(\frac{1}{\varepsilon_{s}}-\frac{1}{\varepsilon_{p}}\right)} \mathrm{d} x .
\end{aligned}
$$

- If $\varepsilon_{s p} \neq 0$,

$$
\begin{aligned}
f_{Q^{(1)}(k)}(q) & =\frac{\sqrt{\pi} e^{-\frac{q}{2 \zeta}\left(\frac{1}{\varepsilon_{s}}+\frac{1}{\varepsilon_{p}}\right)}}{\Gamma\left(N_{s}-k\right) \zeta^{N_{s}-k+1 / 2} \sqrt{\varepsilon_{s} \varepsilon_{p}}}\left(\frac{q}{\varepsilon_{s}-\varepsilon_{p}}\right)^{N_{s}-k-1 / 2} \\
& \times I_{N_{s}-k-1 / 2}\left(\frac{q}{2 \zeta}\left(-\frac{1}{\varepsilon_{s}}+\frac{1}{\varepsilon_{p}}\right)\right),
\end{aligned}
$$

where $I_{n}(\cdot)$ is the modified Bessel function of the first kind. (73) was obtained using [27, (3.383.2.11)].

- If $\varepsilon_{s p}=0$,

$$
f_{Q^{(1)}(k)}(q)=\frac{q^{2\left(N_{s}-k\right)-1} e^{-\frac{q}{\zeta \varepsilon_{s}}}}{\Gamma\left(2\left(N_{s}-k\right)\right)\left(\zeta \varepsilon_{s}\right)^{2\left(N_{s}-k\right)}},
$$

which was obtained using [27, (3.191.1)].

Correspondingly, the CDF of $Q^{(1)}(k)$ is given by $F_{Q^{(1)}(k)}\left(\gamma_{q}\right)$

$$
=\frac{1}{\Gamma\left(N_{s}-k\right)^{2} \varepsilon_{s}^{N_{s}-k}} \int_{0}^{\frac{\gamma_{q}}{\zeta}} x^{N_{s}-k-1} e^{-\frac{x}{\varepsilon_{s}}} \gamma\left(N_{s}-k, \frac{\frac{\gamma_{q}}{\zeta}-x}{\varepsilon_{p}}\right) \mathrm{d} x
$$

$$
= \begin{cases}\frac{\gamma\left(2\left(N_{s}-k, \frac{\gamma_{q}}{\zeta s_{s}}\right)\right)}{\Gamma\left(2\left(N_{s}-k\right)\right)}, & \text { if } \varepsilon_{s p}=0, \\ \frac{\gamma\left(N_{s}-k, \frac{\gamma q}{\zeta s}\right)}{\Gamma\left(N_{s}-k\right)}-\left(\frac{\gamma_{q}}{\zeta \varepsilon_{s}}\right)^{N_{s}-k} e^{-\frac{\gamma q}{\zeta \varepsilon_{p}} \sum_{j=0}^{N_{s}-k-1}} & \\ \times \frac{\left(\frac{\gamma q}{\zeta \varepsilon_{p}}\right)^{j}{ }_{1} F_{1}\left(N_{s}-k ; N_{s}-k+j+1 ; \frac{\gamma q}{\zeta}\left(\frac{1}{\varepsilon_{p}}-\frac{1}{\varepsilon_{s}}\right)\right)}{\Gamma\left(N_{s}-k+j+1\right)}, & \text { if } \varepsilon_{s p} \neq 0,\end{cases}
$$

where (75) was obtained using Table I-(b).

\section{APPENDIX B}

EXPRESSIONS OF $\mathcal{I}_{1}\left(\xi_{s}, \gamma_{t h}\right), \mathcal{I}_{k}\left(\xi_{s}, \gamma_{t h}\right)$ AND $\mathcal{I}_{N_{s}-1}\left(\xi_{s}, \gamma_{t h}\right)$

A. Expression of $\mathcal{I}_{1}\left(\xi_{s}, \gamma_{t h}\right)$

First, we can write $\mathcal{I}_{1}\left(\xi_{s}, \gamma_{t h}\right)$ as

$$
\mathcal{I}_{1}\left(\xi_{s}, \gamma_{t h}\right)=\int_{\frac{\gamma_{t h}}{\xi_{s}}}^{\infty} F_{\gamma_{s}}^{(1)}\left(\xi_{s} y\right) f_{\gamma_{p}}^{(1)}(y) \mathrm{d} y-F_{\gamma_{s}}^{(1)}\left(\gamma_{t h}\right)\left[1-F_{\gamma_{p}}^{(1)}\left(\frac{\gamma_{t h}}{\xi_{s}}\right)\right] \text {. }
$$

For the Rayleigh fading channels, we can show that $\mathcal{I}_{1}\left(\xi_{s}, \gamma_{t h}\right)$ is equal to

$$
\mathcal{I}_{1}\left(\xi_{s}, \gamma_{t h}\right)=\frac{\xi_{s} \varepsilon_{p}}{\xi_{s} \varepsilon_{p}+\varepsilon_{s}} e^{-\gamma_{t h}\left(\frac{1}{\varepsilon_{s}}+\frac{1}{\xi_{s} \varepsilon_{p}}\right)}
$$

\section{B. Expression of $\mathcal{I}_{k}\left(\xi_{s}, \gamma_{t h}\right)$}

Then, we can compute $\mathcal{I}_{k}\left(\xi_{s}, \gamma_{t h}\right)$ as follows

$$
\begin{aligned}
\mathcal{I}_{k}\left(\xi_{s}, \gamma_{t h}\right) & =\int_{\frac{\gamma_{t h}}{\xi_{s}}}^{\infty}\left[\int_{\xi_{s} y-\gamma_{t h}}^{\xi_{s} y} F_{\gamma_{s}}^{(k-1)}\left(-z+\xi_{s} y\right) f_{\gamma_{s}}^{(1)}(z) \mathrm{d} z\right] f_{\gamma_{p}}^{(k)}(y) \mathrm{d} y \\
& -\left[1-F_{\gamma_{p}}^{(k)}\left(\frac{\gamma_{t h}}{\xi_{s}}\right)\right] \int_{0}^{\gamma_{t h}} F_{\gamma_{s}}^{(k-1)}\left(\gamma_{t h}-z\right) f_{\gamma_{s}}^{(1)}(z) \mathrm{d} z \\
& +F_{\gamma_{s}}^{(k-1)}\left(\gamma_{t h}\right) \int_{\frac{\gamma_{t h}}{\xi_{s}}}^{\infty} F_{\gamma_{s}}^{(1)}\left(\xi_{s} y-\gamma_{t h}\right) f_{\gamma_{p}}^{(k)}(y) \mathrm{d} y
\end{aligned}
$$

For the Rayleigh fading channels, $I_{k}\left(\xi_{s}, \gamma_{t h}\right)$ can be written equal to

$$
\begin{aligned}
& \mathcal{I}_{k}\left(\xi_{s}, \gamma_{t h}\right) \\
& =\frac{1}{\Gamma(k) \Gamma(k-1)}\left[\int_{\frac{\gamma_{t h}}{\xi_{s}}}^{\infty}\left[\int_{\xi_{s} y-\gamma_{t h}}^{\xi_{s} y} \gamma\left(k-1, \frac{-z+\xi_{s} y}{\varepsilon_{s}}\right) \frac{e^{-\frac{z}{\varepsilon_{s}}}}{\varepsilon_{s}} \mathrm{~d} z\right]\right. \\
& \times \frac{y^{k-1} e^{-\frac{y}{\varepsilon_{p}}}}{\varepsilon_{p}^{k}} \mathrm{~d} y-\frac{\Gamma\left(k, \frac{\gamma_{t h}}{\xi_{s} \varepsilon_{p}}\right)}{\varepsilon_{s}} \int_{0}^{\gamma_{t h}} \gamma\left(k-1, \frac{\gamma_{t h}-z}{\varepsilon_{s}}\right) e^{-\frac{z}{\varepsilon_{s}}} \mathrm{~d} z \\
& \left.+\frac{\gamma\left(k-1, \frac{\gamma_{t h}}{\varepsilon_{s}}\right)}{\varepsilon_{p}^{k}} \int_{\frac{\gamma_{t h}}{\xi_{s}}}^{\infty}\left(1-e^{-\frac{\xi_{s} y-\gamma_{t h}}{\varepsilon_{s}}}\right) y^{k-1} e^{-\frac{y}{\varepsilon_{p}}} \mathrm{~d} y\right] .
\end{aligned}
$$

Then, using Table I-(a) and the integral equality in [27, (3.381.3)], we can show that

$\mathcal{I}_{k}\left(\xi_{s}, \gamma_{t h}\right)=\frac{e^{-\frac{\gamma_{t h}}{\varepsilon_{s}}}\left(\frac{\gamma_{t h}}{\varepsilon_{s}}\right)^{k-1}}{\Gamma(k)^{2}}\left[\Gamma\left(k, \frac{\gamma_{t h}}{\xi_{s} \varepsilon_{p}}\right)-\frac{e^{\frac{\gamma_{t h}}{\varepsilon_{s}}} \Gamma\left(k, \gamma_{t h}\left(\frac{1}{\varepsilon_{s}}+\frac{1}{\xi_{s} \varepsilon_{p}}\right)\right)}{\varepsilon_{p}^{k}\left(\frac{\xi_{s}}{\varepsilon_{s}}+\frac{1}{\varepsilon_{p}}\right)^{k}}\right]$.

\section{Expression of $\mathcal{I}_{N_{s}-1}\left(\xi_{s}, \gamma_{t h}\right)$}

Finally, we can compute $\mathcal{I}_{N_{s}-1}\left(\xi_{s}, \gamma_{t h}\right)$ as

$\mathcal{I}_{N_{s}-1}\left(\xi_{s}, \gamma_{t h}\right)$

$$
\begin{aligned}
& =\int_{\frac{\gamma_{t h}}{\xi_{s}}}^{\infty}\left[\int_{\xi_{s} y-\gamma_{t h}}^{\xi_{s} y} F_{\gamma_{s}}^{\left(N_{s}-2\right)}\left(-z+\xi_{s} y\right) f_{\gamma_{s}}^{(1)}(z) \mathrm{d} z\right] f_{\gamma_{p}}^{\left(N_{s}-1\right)}(y) \mathrm{d} y \\
& +\int_{0}^{\frac{\gamma_{h b}}{\xi_{s}}}\left[\int_{0}^{\xi_{s} y} F_{\gamma_{s}}^{\left(N_{s}-2\right)}\left(-z+\xi_{s} y\right) f_{\gamma_{s}}^{(1)}(z) \mathrm{d} z\right] f_{\gamma_{p}}^{\left(N_{s}-1\right)}(y) \mathrm{d} y
\end{aligned}
$$




$$
+F_{\gamma_{s}}^{\left(N_{s}-2\right)}\left(\gamma_{t h}\right) \int_{\frac{\gamma_{t h}}{\xi_{s}}}^{\infty} F_{\gamma_{s}}^{(1)}\left(\xi_{s} y-\gamma_{t h}\right) f_{\gamma_{p}}^{\left(N_{s}-1\right)}(y) \mathrm{d} y .
$$

For the Rayleigh fading channels, $I_{N_{s}-1}\left(\xi_{s}, \gamma_{t h}\right)$ can be shown equal to

$$
\begin{aligned}
& \mathcal{I}_{N_{s}-1}\left(\xi_{s}, \gamma_{t h}\right) \\
& =\frac{\gamma\left(N_{s}-2, \frac{\gamma_{t h}}{\varepsilon_{s}}\right) \Gamma\left(N_{s}-1, \frac{\gamma_{t h}}{\xi_{s} \varepsilon_{p}}\right)}{\Gamma\left(N_{s}-2\right) \Gamma\left(N_{s}-1\right)}-\frac{\left(\frac{\gamma_{t h}}{\varepsilon_{s}}\right)^{N_{s}-2} \Gamma\left(N_{s}-1, \gamma_{t h}\left(\frac{1}{\xi_{s} \varepsilon_{p}}+\frac{1}{\varepsilon_{s}}\right)\right)}{\Gamma\left(N_{s}-1\right)^{2} \varepsilon_{p}^{N_{s}-1}\left(\frac{1}{\varepsilon_{p}}+\frac{\xi_{s}}{\varepsilon_{s}}\right)^{N_{s}-1}} \\
& +\frac{1}{\Gamma\left(N_{s}-1\right)^{2}} \int_{0}^{\frac{\gamma_{t h}}{\xi_{s} \varepsilon_{p}}} \gamma\left(N_{s}-1, u \frac{\xi_{s} \varepsilon_{p}}{\varepsilon_{s}}\right) u^{N_{s}-2} e^{-u} \mathrm{~d} u,
\end{aligned}
$$

which was obtained using Table I-(a) and the integral equality of the upper incomplete Gamma function in [27, (3.381.3)]. Then, we use the sum representation of the lower incomplete Gamma function in $[27,(8.352 .1)]$ and the integral equality of the lower incomplete Gamma function in [27, (3.381.1)], to get

$$
\begin{aligned}
\mathcal{I}_{N_{s}-1}\left(\xi_{s}, \gamma_{t h}\right)= & 1-\frac{\Gamma\left(N_{s}-2, \frac{\gamma_{t h}}{\varepsilon_{s}}\right) \Gamma\left(N_{s}-1, \frac{\gamma_{t h}}{\xi_{s} \varepsilon_{p}}\right)}{\Gamma\left(N_{s}-2\right) \Gamma\left(N_{s}-1\right)} \\
& -\frac{\left(\frac{\gamma_{t h}}{\varepsilon_{s}}\right)^{N_{s}-2} \Gamma\left(N_{s}-1, \gamma_{t h}\left(\frac{1}{\xi_{s} \varepsilon_{p}}+\frac{1}{\varepsilon_{s}}\right)\right)}{\Gamma\left(N_{s}-1\right)^{2} \varepsilon_{p}^{N_{s}-1}\left(\frac{1}{\varepsilon_{p}}+\frac{\xi_{s}}{\varepsilon_{s}}\right)^{N_{s}-1}} \\
& -\sum_{m=0}^{N_{s}-2} \frac{\left(\frac{\xi_{s} \varepsilon_{p}}{\varepsilon_{s}}\right)^{m} \gamma\left(N_{s}+m-1, \gamma_{t h}\left(\frac{1}{\varepsilon_{s}}+\frac{1}{\xi_{s} \varepsilon_{p}}\right)\right)}{\Gamma\left(N_{s}-1\right) \Gamma(m+1)\left(\frac{\xi_{s} \varepsilon_{p}}{\varepsilon_{s}}+1\right)^{N_{s}+m-1} .}
\end{aligned}
$$

$$
\begin{gathered}
\text { APPENDIX C } \\
\text { ExPRESSIONS OF } \mathcal{J}_{1}\left(\gamma_{q}, \gamma_{t h}\right), \mathcal{J}_{k}\left(\gamma_{q}, \gamma_{t h}\right) \text { AND } \mathcal{J}_{N_{s}-1}\left(\gamma_{q}, \gamma_{t h}\right)
\end{gathered}
$$

A. Expression of $\mathcal{J}_{1}\left(\gamma_{q}, \gamma_{\text {th }}\right)$

Let us start by expressing $\mathcal{J}_{1}\left(\gamma_{q}, \gamma_{t h}\right)$ :

$$
\begin{aligned}
\mathcal{J}_{1}\left(\gamma_{q}, \gamma_{t h}\right) & =\operatorname{Prob}\left(Q(1) \leq \gamma_{q} \& \bar{\Gamma}_{s}(2)<\gamma_{t h}\right) \\
& = \begin{cases}\mathcal{G}_{0}, & \text { if } \delta_{q, t h} \leq 0, \\
\mathcal{G}_{1}(1), & \text { if } \delta_{q, t h}>0,\end{cases}
\end{aligned}
$$

where $\mathcal{G}_{0}$ is defined in (86) for $\delta_{q, t h} \leq 0$, and $\mathcal{G}_{1}(k)$ is defined in (87) for $k=1, \ldots, N_{s}-1$ and $\delta_{q, t h}>0$.

For the Rayleigh fading channels, we use some mathematical manipulations and Table I to show that $\mathcal{J}_{1}\left(\gamma_{q}, \gamma_{t h}\right)$ is expressed depending on the sign of $\delta_{q, t h}$ and the value of $\varepsilon$ either equal to zero or not, as follows.

- If $\delta_{q, t h} \leq 0$, we use Table I-(c) to show that (85) is equivalently equal to

$$
\begin{aligned}
& \mathcal{J}_{1}\left(\gamma_{q}, \gamma_{t h}\right) \\
& =\frac{1}{\Gamma\left(N_{s}-1\right)^{2} \varepsilon_{p}^{N_{s}-1}} \int_{0}^{\frac{\gamma_{q}}{\zeta}} y^{N_{s}-2} e^{-\frac{y}{\varepsilon_{p}}} \gamma\left(N_{s}-1, \frac{\frac{\gamma_{q}}{\zeta}-y}{\varepsilon_{s}}\right) \mathrm{d} y .
\end{aligned}
$$

Then, using Table I-(b), we show that (88) is expressed, depending on $\varepsilon_{s p}$, either zero or not, as follows

$$
\mathcal{J}_{1}\left(\gamma_{q}, \gamma_{t h}\right)= \begin{cases}\psi\left(1, \frac{\gamma_{q}}{\zeta \varepsilon_{s}}\right), & \text { if } \varepsilon_{s p}=0 \\ \varphi\left(1, \frac{\gamma_{q}}{\zeta \varepsilon_{p}}\right)-\chi\left(1, \frac{\gamma_{q}}{\zeta}\right), & \text { if } \varepsilon_{s p} \neq 0\end{cases}
$$

with $\psi(k, x)=\frac{\gamma\left(2\left(N_{s}-k\right), x\right)}{\Gamma\left(2\left(N_{s}-k\right)\right)}, \varphi(k, x)=\frac{\gamma\left(N_{s}-k, x\right)}{\Gamma\left(N_{s}-k\right)}$, and $\chi(k, x)=\left(\frac{x}{\varepsilon_{p}}\right)^{N_{s}-k} e^{-\frac{x}{\varepsilon_{s}}} \sum_{j=0}^{N_{s}-k-1} \frac{\left(\frac{x}{\varepsilon_{s}}\right)_{1}^{j} F_{1}\left(N_{s}-k ; N_{s}-k+j+1 ;-\varepsilon_{s p} x\right)}{\Gamma\left(N_{s}-k+j+1\right)}$.

- If $\delta_{q, t h}>0$, we use Table I-(c) and Table I-(d) to show that (85) is equivalently equal to

$$
\begin{aligned}
& \mathcal{J}_{1}\left(\gamma_{q}, \gamma_{t h}\right)=\frac{e^{-\frac{\delta_{q, t h}}{\varepsilon_{s}}} \gamma\left(N_{s}-1, \frac{\gamma_{t h}}{\varepsilon_{s}}\right)}{\Gamma\left(N_{s}-1\right)^{2} \varepsilon_{p}^{N_{s}-1}} \int_{0}^{\delta_{q, t h}} y^{N_{s}-2} e^{-\varepsilon_{s p} y} \mathrm{~d} y \\
& +\frac{\gamma\left(N_{s}-2, \frac{\gamma_{t h}}{\varepsilon_{s}}\right)}{\varepsilon_{s} \Gamma\left(N_{s}-1\right) \Gamma\left(N_{s}-2\right)} \int_{0}^{\delta_{q, t h}} e^{-\frac{z}{\varepsilon_{s}}} \gamma\left(N_{s}-1, \frac{\delta_{q, t h}-z}{\varepsilon_{p}}\right) \mathrm{d} z \\
& -\frac{1}{\Gamma\left(N_{s}-1\right)^{2} \varepsilon_{p}^{N_{s}-1}} \int_{0}^{\delta_{q, t h}} y^{N_{s}-2} e^{-\frac{y}{\varepsilon_{p}}} \gamma\left(N_{s}-1, \frac{\delta_{q, t h}-y}{\varepsilon_{s}}\right) \mathrm{d} y \\
& +\frac{1}{\Gamma\left(N_{s}-1\right)^{2} \varepsilon_{p}^{N_{s}-1}} \int_{0}^{\frac{\gamma \gamma_{q}}{\zeta}} y^{N_{s}-2} e^{-\frac{y}{\varepsilon_{p}}} \gamma\left(N_{s}-1, \frac{\frac{\gamma_{q}}{\zeta}-y}{\varepsilon_{s}}\right) \mathrm{d} y .
\end{aligned}
$$

Then, using Table I-(b) and Table I-(c), we show that (90) is expressed, depending on $\varepsilon_{s p}$, either zero or not, as follows

$$
\mathcal{J}_{1}\left(\gamma_{q}, \gamma_{t h}\right)= \begin{cases}\Phi_{1}\left(1, \gamma_{t h}, \delta_{q, t h}\right), & \text { if } \varepsilon_{s p}=0, \\ \Omega_{1}\left(1, \gamma_{t h}, \delta_{q, t h}\right), & \text { if } \varepsilon_{s p} \neq 0,\end{cases}
$$

where $\quad \Phi_{1}\left(k, \gamma_{t h}, \delta_{q, t h}\right), \quad$ and $\quad \Omega_{1}\left(k, \gamma_{t h}, \delta_{q, t h}\right)$ are expressed in (92) and (93) for $k=$ $1, \ldots, N_{s}-1, \quad$ respectively, with $\omega(k, x, y)=$ $\varphi\left(k+1, \frac{x}{\varepsilon_{s}}\right)\left[1-e^{-\frac{y}{\varepsilon_{s}}}-\frac{e^{-\frac{y}{\varepsilon_{s}}}}{\varepsilon_{s}} \sum_{j=0}^{N_{s}-k-1} \frac{\gamma\left(j+1, \varepsilon_{s p} y\right)}{\Gamma(j+1) \varepsilon_{p}^{j} \varepsilon_{s p}^{j+1}}\right]$.

\section{B. Expression of $\mathcal{J}_{k}\left(\gamma_{q}, \gamma_{t h}\right)$}

Then, let us compute $\mathcal{J}_{k}\left(\gamma_{q}, \gamma_{t h}\right)$

$$
\mathcal{J}_{k}\left(\gamma_{q}, \gamma_{t h}\right)=\operatorname{Prob}\left(Q(k) \leq \gamma_{q} \& \bar{\Gamma}_{s}(k+1)<\gamma_{\text {th }} \leq \bar{\Gamma}_{s}(k)\right)
$$

$$
= \begin{cases}\mathcal{G}_{1}(k)+\mathcal{G}_{2}\left(k, \delta_{q, t h}, \frac{\gamma_{q}}{\zeta}\right), & \text { if } 0<\delta_{q, t h}<\gamma_{t h}, \\ \mathcal{G}_{1}(k)+\mathcal{G}_{2}\left(k, \gamma_{t h}, \delta_{q, t h}\right), & \text { if } \gamma_{t h} \leq \delta_{q, t h},\end{cases}
$$

where $\mathcal{G}_{2}(k, a, b)$ is defined in (96) for $k=2, \ldots, N_{s}-2$, $a \leq \gamma_{t h}$, and $b \leq \frac{\gamma_{q}}{\zeta}$.

For the Rayleigh fading channels, we use some mathematical manipulations and Table I to show that $\mathcal{J}_{k}\left(\gamma_{q}, \gamma_{t h}\right)$ is expressed depending on $\delta_{q, t h}, \gamma_{t h}$ and $\varepsilon_{s p}$, as follows:

- If $0<\delta_{q, t h}<\gamma_{t h}$, we use Table I-(c) and Table I-(d) to show that (95) is equivalently equal to

$$
\begin{aligned}
& \mathcal{J}_{k}\left(\gamma_{q}, \gamma_{t h}\right)=\frac{e^{-\frac{\delta_{q, t h}}{\varepsilon_{s}}} \gamma\left(N_{s}-k, \frac{\gamma_{t h}}{\varepsilon_{s}}\right)}{\varepsilon_{p}^{N_{s}-k} \Gamma\left(N_{s}-k\right)^{2}} \int_{0}^{\delta_{q, t h}} y^{N_{s}-k-1} e^{-\varepsilon_{s p} y} \mathrm{~d} y \\
& +\frac{\gamma\left(N_{s}-k-1, \frac{\gamma_{t h}}{\varepsilon_{s}}\right)}{\varepsilon_{s} \Gamma\left(N_{s}-k\right) \Gamma\left(N_{s}-k-1\right)} \int_{0}^{\delta_{q, t h}} e^{-\frac{z}{\varepsilon_{s}}} \gamma\left(N_{s}-k, \frac{\delta_{q, t h}-z}{\varepsilon_{p}}\right) \mathrm{d} z \\
& -\frac{e^{-\frac{\delta_{q, t h}}{\varepsilon_{s}}}}{\varepsilon_{p}^{N_{s}-k} \Gamma\left(N_{s}-k\right)^{2}} \int_{0}^{\delta_{q, t h}} y^{N_{s}-k-1} e^{-\frac{y}{\varepsilon_{p}}} \gamma\left(N_{s}-k, \frac{\gamma_{t h}-y}{\varepsilon_{s}}\right) \mathrm{d} y \\
& -\frac{\gamma\left(N_{s}-k, \frac{\delta_{q, t h}}{\varepsilon_{p}}\right)}{\Gamma\left(N_{s}-k\right)^{2}}\left[\gamma\left(N_{s}-k, \frac{\gamma_{t h}}{\varepsilon_{s}}\right)-e^{-\frac{\delta_{q, t h}}{\varepsilon_{s}}} \gamma\left(N_{s}-k, \frac{\gamma_{t h}-\delta_{q, t h}}{\varepsilon_{s}}\right)\right]
\end{aligned}
$$




$$
\begin{aligned}
& \mathcal{G}_{0}= \int_{0}^{\frac{\gamma_{q}}{\zeta}} f_{\gamma_{s}}^{(1)}(z)\left[\int_{0}^{\frac{\gamma_{q}}{\zeta}-z} f_{\gamma_{p}}^{\left(N_{s}-1\right)}(y) F_{\gamma_{s}}^{\left(N_{s}-2\right)}\left(\frac{\gamma_{q}}{\zeta}-z-y\right) \mathrm{d} y\right] \mathrm{d} z, \\
& \mathcal{G}_{1}(k)=\left\{\begin{array}{l}
\int_{0}^{\frac{\gamma_{q}}{\zeta}} f_{\gamma_{s}}^{(1)}(z)\left[\int_{0}^{\frac{\gamma_{q}}{\zeta}-z} f_{\gamma_{p}}^{\left(N_{s}-1\right)}(y) F_{\gamma_{s}}^{\left(N_{s}-2\right)}\left(\frac{\gamma_{q}}{\zeta}-z-y\right) \mathrm{d} y\right] \mathrm{d} z+F_{\gamma_{s}}^{\left(N_{s}-2\right)}\left(\gamma_{t h}\right)\left[\int_{0}^{\delta_{q, t h}} f_{\gamma_{s}}^{(1)}(z) F_{\gamma_{p}}^{\left(N_{s}-1\right)}\left(\delta_{q, t h}-z\right) \mathrm{d} z\right] \\
-\int_{0}^{\delta_{q, t h}} f_{\gamma_{s}}^{(1)}(z)\left[\int_{0}^{\delta_{q, t h}-z} f_{\gamma_{p}}^{\left(N_{s}-1\right)}(y) F_{\gamma_{s}}^{\left(N_{s}-2\right)}\left(\frac{\gamma_{q}}{\zeta}-z-y\right) \mathrm{d} y\right] \mathrm{d} z, \\
F_{\gamma_{s}}^{\left(N_{s}-k-1\right)}\left(\gamma_{t h}\right)\left[\int_{0}^{\delta_{q, t h}} f_{\gamma_{s}}^{(1)}(z) F_{\gamma_{p}}^{\left(N_{s}-k\right)}\left(\delta_{q, t h}-z\right) \mathrm{d} z\right]-\int_{0}^{\delta_{q, t h}} f_{\gamma_{s}}^{(1)}(z)\left[\int_{0}^{\delta_{q, t h}-z} f_{\gamma_{p}}^{\left(N_{s}-k\right)}(y) F_{\gamma_{s}}^{\left(N_{s}-k-1\right)}\left(\frac{\gamma_{q}}{\zeta}-z-y\right) \mathrm{d} y\right] \mathrm{d} z, \text { if } k=2, \ldots, N_{s}-2, \\
\int_{0}^{\delta_{q, t h}} f_{\gamma_{p}}^{(1)}(y) F_{\gamma_{s}}^{(1)}\left(\frac{\gamma_{q}}{\zeta}-y\right) \mathrm{d} y-F_{\gamma_{p}}^{(1)}\left(\delta_{q, t h}\right) F_{\gamma_{s}}^{(1)}\left(\gamma_{t h}\right),
\end{array} \text { if } k=N_{s}-1 .\right.
\end{aligned}
$$

$\Phi_{1}\left(k, \gamma_{t h}, \delta_{q, t h}\right)= \begin{cases}\psi\left(1, \frac{\gamma_{q}}{\zeta \varepsilon_{s}}\right)-\psi\left(1, \frac{\delta_{q, t h}}{\varepsilon_{s}}\right)+\varphi\left(2, \frac{\gamma_{t h}}{\varepsilon_{s}}\right) \varphi\left(0, \frac{\delta_{q, t h}}{\varepsilon_{s}}\right)+\frac{\left(\frac{\delta_{q, t h}}{\varepsilon_{s}}\right)^{N_{s}-1} e^{-\frac{\delta_{q, t h}}{\varepsilon_{s}}}}{\Gamma\left(N_{s}\right)} \varphi\left(1, \frac{\gamma_{t h}}{\varepsilon_{s}}\right), & \text { if } k=1, \\ \varphi\left(k+1, \frac{\gamma_{t h}}{\varepsilon_{s}}\right) \varphi\left(k-1, \frac{\delta_{q, t h}}{\varepsilon_{s}}\right)-\varphi\left(k, \frac{\delta_{q, t h}}{\varepsilon_{s}}\right) \varphi\left(k, \frac{\gamma_{t h}}{\varepsilon_{s}}\right)+e^{-\frac{\delta_{q, t h}}{\varepsilon_{s}} \frac{\left(\frac{\delta_{q, t h}}{\varepsilon_{s}}\right)^{N_{s}-k} \varphi\left(k, \frac{\gamma_{t h}}{\varepsilon_{s}}\right)}{\Gamma\left(N_{s}-k+1\right)}-e^{-\frac{\delta_{q, t h}}{\varepsilon_{s}}} \psi\left(k, \frac{\gamma_{t h}}{\varepsilon_{s}}\right),} & \text { if } k=2, \ldots, N_{s}-2, \\ e^{-\frac{\gamma_{t h}}{\varepsilon_{s}}}-e^{-\frac{\gamma_{q}}{\varepsilon_{s}}}\left(1+\frac{\delta_{q, t h}}{\varepsilon_{s}}\right), & \text { if } k=N_{s}-1,\end{cases}$

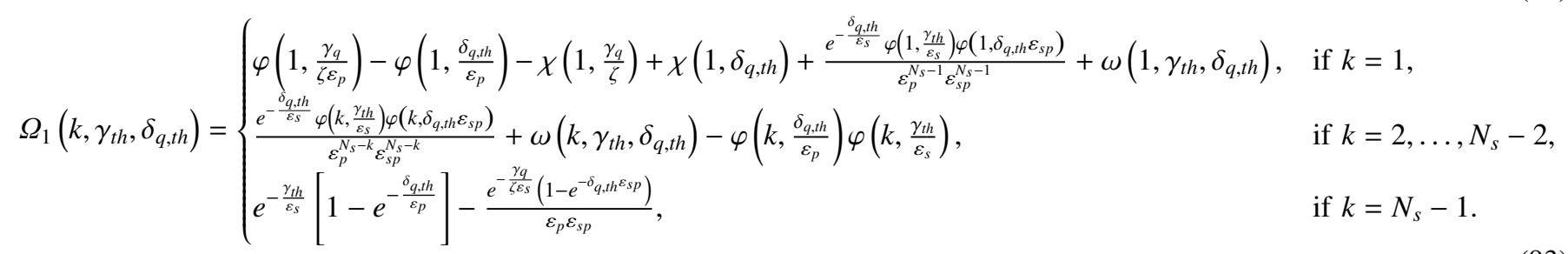

$$
\begin{aligned}
\mathcal{G}_{2}(k, a, b) & =\int_{0}^{a} f_{\gamma_{s}}^{(1)}(z)\left[\int_{0}^{\delta_{q, t h}} f_{\gamma_{p}}^{\left(N_{s}-k\right)}(y) F_{\gamma_{s}}^{\left(N_{s}-k-1\right)}\left(\frac{\gamma_{q}}{\zeta}-z-y\right) \mathrm{d} y\right] \mathrm{d} z+\int_{0}^{b} f_{\gamma_{s}}^{(1)}(z)\left[\int_{0}^{\frac{\gamma_{q}}{\zeta}-z} f_{\gamma_{p}}^{\left(N_{s}-k\right)}(y) F_{\gamma_{s}}^{\left(N_{s}-k-1\right)}\left(\frac{\gamma_{q}}{\zeta}-z-y\right) \mathrm{d} y\right] \mathrm{d} z \\
& -\int_{0}^{\gamma_{t h}} f_{\gamma_{s}}^{(1)}(z)\left[\int_{0}^{\frac{\gamma_{q}}{\zeta}-z} f_{\gamma_{p}}^{\left(N_{s}-k\right)}(y) F_{\gamma_{s}}^{\left(N_{s}-k-1\right)}\left(\frac{\gamma_{q}}{\zeta}-z-y\right) \mathrm{d} y\right] \mathrm{d} z-F_{\gamma_{p}}^{\left(N_{s}-k\right)}\left(\delta_{q, t h}\right)\left[\int_{0}^{a} f_{\gamma_{s}}^{(1)}(z) F_{\gamma_{s}}^{\left(N_{s}-k-1\right)}\left(\gamma_{t h}-z\right) \mathrm{d} z\right] .
\end{aligned}
$$

$$
+\frac{e^{-\frac{\gamma_{t h}}{\varepsilon_{s}}}}{\varepsilon_{p}^{N_{s}-k} \Gamma\left(N_{s}-k\right)^{2}} \int_{0}^{\delta_{q, t h}} y^{N_{s}-k-1} e^{-\frac{y}{\varepsilon_{p}}} \gamma\left(N_{s}-k, \frac{\delta_{q, t h}-y}{\varepsilon_{s}}\right) \mathrm{d} y .
$$

Then, we use Table I-(b), Table I-(c) and Table I-(e) and we show that (97) is expressed, depending on $\varepsilon_{s p}$, either zero or not, as follows

$\mathcal{J}_{k}\left(\gamma_{q}, \gamma_{t h}\right)=\left\{\begin{array}{l}\Phi_{1}\left(k, \gamma_{t h}, \delta_{q, t h}\right)+\Phi_{2}\left(k, \gamma_{t h}, \delta_{q, t h}\right), \text { if } \varepsilon_{s p}=0 \\ \Omega_{1}\left(k, \gamma_{t h}, \delta_{q, t h}\right)+\Omega_{2}\left(k, \gamma_{t h}, \delta_{q, t h}\right), \text { if } \varepsilon_{s p} \neq 0\end{array}\right.$

where $\quad \Phi_{2}\left(k, \gamma_{t h}, \delta_{q, t h}\right) \quad$ and $\quad \Omega_{2}\left(k, \gamma_{t h}, \delta_{q, t h}\right)$ are given in (99) and in (100) for $k=$ $2, \ldots, N_{s}-2$, respectively, with $v(k, x, y)=$ $\frac{\left(\frac{x}{\varepsilon_{s}}\right)^{N_{s}-k-1} e^{-\frac{x+y}{\varepsilon_{s}}}}{\Gamma\left(N_{s}-k\right)} \sum_{j=0}^{N_{s}-k-1} \frac{\left(\frac{y-x}{\varepsilon_{s}}\right)^{j+1}}{\Gamma(j+2)}{ }_{2} F_{1}\left(1,-N_{s}+k+1 ; j+2 ;-\frac{y-x}{x}\right)$, and $\kappa(k, x, z)=\frac{e^{-\frac{x+z}{\varepsilon_{s}}}}{\varepsilon_{p}^{N_{s}-k} \Gamma\left(N_{s}-k\right)} \sum_{j=0}^{N_{s}-k-1} \sum_{l=0}^{j} \frac{\left(\begin{array}{l}j \\ l\end{array}\right)^{z^{j-l}(-1)^{l} \gamma\left(N_{s}-k+l, x \varepsilon_{s p}\right)}}{\varepsilon_{s p}^{N_{s}-k+l} \Gamma(j+1) \varepsilon_{s}^{j}}$,
for $x \leq y$.

- If $\gamma_{t h} \leq \delta_{q, t h}$, we use Table I-(c) and Table I-(d) to show that (95) is equivalently equal to

$$
\begin{aligned}
& \mathcal{J}_{k}\left(\gamma_{q}, \gamma_{t h}\right)=\frac{e^{-\frac{\delta_{q, t h}}{\varepsilon_{s}}} \gamma\left(N_{s}-k, \frac{\gamma_{t h}}{\varepsilon_{s}}\right)}{\varepsilon_{p}^{N_{s}-k} \Gamma\left(N_{s}-k\right)^{2}} \int_{0}^{\delta_{q, t h}} y^{N_{s}-k-1} e^{-\varepsilon_{s p} y} \mathrm{~d} y \\
& +\frac{\gamma\left(N_{s}-k-1, \frac{\gamma_{t h}}{\varepsilon_{s}}\right)}{\varepsilon_{s} \Gamma\left(N_{s}-k\right) \Gamma\left(N_{s}-k-1\right)} \int_{0}^{\delta_{q, t h}} e^{-\frac{z}{\varepsilon_{s}}} \gamma\left(N_{s}-k, \frac{\delta_{q, t h}-z}{\varepsilon_{p}}\right) \mathrm{d} z \\
& -\frac{e^{-\frac{\delta_{q, t h}}{\varepsilon_{s}}}}{\varepsilon_{p}^{N_{s}-k} \Gamma\left(N_{s}-k\right)^{2}} \int_{0}^{\gamma_{t h}} y^{N_{s}-k-1} e^{-\frac{y}{\varepsilon_{p}}} \gamma\left(N_{s}-k, \frac{\gamma_{t h}-y}{\varepsilon_{s}}\right) \mathrm{d} y \\
& -\frac{\gamma\left(N_{s}-k, \frac{\delta_{q, t h}}{\varepsilon_{p}}\right) \gamma\left(N_{s}-k, \frac{\gamma_{t h}}{\varepsilon_{s}}\right)}{\Gamma\left(N_{s}-k\right)^{2}} .
\end{aligned}
$$

Then, using Table I-(b), and Table I-(c), we show that (101) is expressed, depending on $\varepsilon_{s p}$, either zero or not,

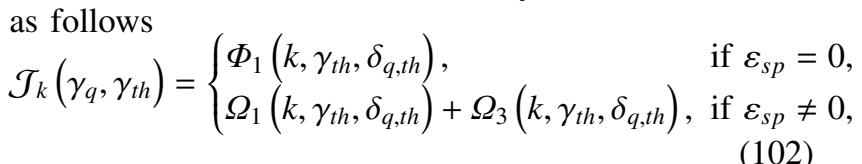

where $\Omega_{3}\left(k, \gamma_{t h}, \delta_{q, t h}\right)$ is given in (103), for $k=2, \ldots, N_{s}-$ 


$$
\begin{aligned}
& \Phi_{2}\left(k, \gamma_{t h}, \delta_{q, t h}\right)=e^{-\frac{\delta_{q, t h}}{\varepsilon_{s}}} \varphi\left(k, \frac{\gamma_{t h}}{\varepsilon_{s}}\right)+e^{-\frac{\delta_{q, t h}}{\varepsilon_{s}}} \varphi\left(k, \frac{\delta_{q, t h}}{\varepsilon_{s}}\right)\left(\varphi\left(k, \frac{\gamma_{t h}-\delta_{q, t h}}{\varepsilon_{s}}\right)-1\right)-v\left(k, \delta_{q, t h}, \gamma_{t h}\right)+e^{-\frac{\gamma_{t h}}{\varepsilon_{s}}} \psi\left(k, \frac{\delta_{q, t h}}{\varepsilon_{s}}\right), \\
& \Omega_{2}\left(k, \gamma_{t h}, \delta_{q, t h}\right)=\kappa\left(k, \delta_{q, t h}, \gamma_{t h}\right)-e^{-\frac{\gamma_{t h}}{\varepsilon_{s}}} \chi\left(k, \delta_{q, t h}\right)+e^{-\frac{\delta_{q, t h}}{\varepsilon_{s}}}\left[\varphi\left(k, \frac{\delta_{q, t h}}{\varepsilon_{p}}\right) \varphi\left(k, \frac{\gamma_{t h}-\delta_{q, t h}}{\varepsilon_{s}}\right)-\varphi\left(k, \frac{\delta_{q, t h}}{\varepsilon_{p}}\right)\right]+e^{-\frac{\gamma_{t h}}{\varepsilon_{s}}} \varphi\left(k, \frac{\delta_{q, t h}}{\varepsilon_{p}}\right)
\end{aligned}
$$

$$
\Omega_{3}\left(k, \gamma_{t h}, \delta_{q, t h}\right)=-e^{-\frac{\delta_{q, t h}}{\varepsilon_{s}}} \varphi\left(k, \frac{\gamma_{t h}}{\varepsilon_{p}}\right)+e^{-\frac{\gamma_{q}}{\xi_{s}}} \chi\left(k, \gamma_{t h}\right)
$$

2.

\section{Expression of $\mathcal{J}_{N_{s}-1}\left(\gamma_{q}, \gamma_{t h}\right)$}

Finally, we can compute $\mathcal{J}_{N_{s}-1}\left(\gamma_{q}, \gamma_{t h}\right)$ and show that it is expressed, only if $\delta_{q, t h}>0$, as

$$
\begin{aligned}
\mathcal{J}_{N_{s}-1}\left(\gamma_{q}, \gamma_{t h}\right) & =\operatorname{Prob}\left(Q\left(N_{s}-1\right) \leq \gamma_{q} \& \bar{\Gamma}_{s}\left(N_{s}-1\right) \geq \gamma_{t h}\right) \\
& =\mathcal{G}_{1}\left(N_{s}-1\right) .
\end{aligned}
$$

For the Rayleigh fading channels, $\mathcal{J}_{N_{s}-1}\left(\gamma_{q}, \gamma_{t h}\right)$ can be rewritten depending on the value $\varepsilon_{s p}$ as follows:

$$
\mathcal{J}_{N_{s}-1}\left(\gamma_{q}, \gamma_{t h}\right)= \begin{cases}\Phi_{1}\left(N_{s}-1, \gamma_{t h}, \delta_{q, t h}\right), & \text { if } \varepsilon_{s p}=0 \\ \Omega_{1}\left(N_{s}-1, \gamma_{t h}, \delta_{q, t h}\right), & \text { if } \varepsilon_{s p} \neq 0\end{cases}
$$

\section{REFERENCES}

[1] J. Mitola and J. Maguire, G.Q., "Cognitive radio: Making software radios more personal," IEEE Personal Communications, vol. 6, no. 4, pp. 1318, August 1999.

[2] A. Ghasemi and E. Sousa, "Fundamental limits of spectrum-sharing in fading environments," IEEE Transactions on Wireless Communications, vol. 6, no. 2, pp. 649-658, February 2007.

[3] L. Musavian and S. Aissa, "Fundamental capacity limits of cognitive radio in fading environments with imperfect channel information," IEEE Transactions on Communications, vol. 57, no. 11, pp. 3472-3480, November 2009.

[4] R. Manna, R. H. Louie, Y. Li, and B. Vucetic, "Cooperative spectrum sharing in cognitive radio networks with multiple antennas," IEEE Transactions on Signal Processing, vol. 59, no. 11, pp. 5509-5522, November 2011.

[5] K. Tourki, F. Khan, K. Qaraqe, H.-C. Yang, and M.-S. Alouini, "Exact performance analysis of MIMO cognitive radio systems using transmit antenna selection," IEEE Journal on Selected Areas in Communications, vol. 32, no. 3, pp. 425-438, Mars 2014.

[6] R. Duan, M. Elmusrati, R. Jantti, and R. Virrankoski, "Capacity for spectrum sharing cognitive radios with MRC diversity at the secondary receiver under asymmetric fading," in IEEE Global Telecommunications Conference (GLOBECOM'2010), Miami, Florida, USA, December 2010, pp. 1-5.

[7] K. Hamdi, W. Zhang, and K. B. Letaief, "Opportunistic spectrum sharing in cognitive MIMO wireless networks," IEEE Transactions on Wireless Communications, vol. 8, no. 8, pp. 4098-4109, August 2009.

[8] L. Varshney, "Transporting information and energy simultaneously," in IEEE International Symposium on Information Theory (ISIT'2008), Toronto, Canada, July 2008, pp. 1612-1616.

[9] P. Grover and A. Sahai, "Shannon meets Tesla: Wireless information and power transfer," in IEEE International Symposium on Information Theory Proceedings (ISIT'2010), Austin, Texas, USA, June 2010, pp. 2363-2367.

[10] R. Zhang and C. K. Ho, "MIMO broadcasting for simultaneous wireless information and power transfer," IEEE Transactions on Wireless Communications, vol. 12, no. 5, pp. 1989-2001, May 2013.

[11] A. Nasir, X. Zhou, S. Durrani, and R. Kennedy, "Relaying protocols for wireless energy harvesting and information processing," IEEE Transactions on Wireless Communications, vol. 12, no. 7, pp. 3622-3636, July 2013.
[12] I. Krikidis, S. Sasaki, S. Timotheou, and Z. Ding, "A low complexity antenna switching for joint wireless information and energy transfer in MIMO relay channels," IEEE Transactions on Communications, vol. 62, no. 5, pp. 1577-1587, May 2014.

[13] F. Benkhelifa, A. S. Salem, and M. S. Alouini, "Rate maximization in MIMO Decode-and-Forward communications with an EH relay and possibly imperfect CSI," IEEE Transactions on Communications, vol. 64, no. 11, pp. 4534-4549, Nov. 2016.

[14] F. Benkhelifa and M. S. Alouini, "Precoding design of MIMO Amplifyand-Forward communication system with an energy harvesting relay and possibly imperfect CSI," IEEE Access, vol. 5, pp. 578-594, 2017.

[15] S. Maghsudi and E. Hossain, "Distributed user association in energy harvesting dense small cell networks: A mean-field multi-armed bandit approach," IEEE Access, vol. 5, pp. 3513-3523, 2017.

[16] A. Ghazanfari, H. Tabassum, and E. Hossain, "Ambient RF energy harvesting in ultra-dense small cell networks: Performance and tradeoffs," IEEE Wireless Communications, vol. 23, no. 2, pp. 38-45, April 2016.

[17] G. Lee, W. Saad, M. Bennis, A. Mehbodniya, and F. Adachi, "Online ski rental for ON/OFF scheduling of energy harvesting base stations," IEEE Transactions on Wireless Communications, vol. 16, no. 5, pp. 29762990, May 2017.

[18] S. Lee, R. Zhang, and K. Huang, "Opportunistic wireless energy harvesting in cognitive radio networks," IEEE Transactions on Wireless Communications, vol. 12, no. 9, pp. 4788-4799, September 2013.

[19] Z. Yang, Z. Ding, P. Fan, and G. K. Karagiannidis, "Outage performance of cognitive relay networks with wireless information and power transfer," IEEE Transactions on Vehicular Technology, vol. 65, no. 5, pp. 3828-3833, May 2016.

[20] X. Lu, W. Xu, S. Li, J. Lin, and Z. He, "Simultaneous information and power transfer for relay-assisted cognitive radio networks," in IEEE International Conference on Communications Workshops (ICC'2014), Sydney, Australia, June 2014, pp. 331-336.

[21] Z. Wang, Z. Chen, L. Luo, Z. Hu, B. Xia, and H. Liu, "Outage analysis of cognitive relay networks with energy harvesting and information transfer," in IEEE International Conference on Communications (ICC'2014), Sydney, Australia, June 2014, pp. 4348-4353.

[22] S. Yin, E. Zhang, Z. Qu, L. Yin, and S. Li, "Optimal cooperation strategy in cognitive radio systems with energy harvesting," IEEE Transactions on Wireless Communications, vol. 13, no. 9, pp. 4693-4707, September 2014.

[23] V. Rakovic, D. Denkovski, Z. Hadzi-Velkov, and L. Gavrilovska, "Optimal time sharing in underlay cognitive radio systems with RF energy harvesting," in IEEE International Conference on Communications (ICC'2015), London, UK, June 2015, pp. 7689-7694.

[24] L. Sibomana, H. J. Zepernick, and H. Tran, "Wireless information and power transfer in an underlay cognitive radio network," in 8th International Conference on Signal Processing and Communication Systems (ICSPCS'2014), Gold Coast, Australia, December 2014, pp. $1-7$.

[25] F. Benkhelifa, K. Tourki, and M. S. Alouini, "Simultaneous wireless information and power transfer for spectrum sharing in cognitive radio communication systems," in IEEE International Conference on Communications Workshops (ICC'2016), Kuala Lumpur, Malaysia, May 2016, pp. 676-681.

[26] H.-C. Yang and M. S. Alouini, "MRC and GSC diversity combining with an output threshold," IEEE Transactions on Vehicular Technology, vol. 54, no. 3, pp. 1081-1090, May 2005.

[27] I. S. Gradshteyn and I. M. Ryzhik, Table of Integrals, Series, and Products, 5th ed. San Diego, CA: Academic, 1994. 
Table I: Expressions of useful integrals.

\begin{tabular}{|c|c|}
\hline (a) & $\int_{0}^{a} \gamma(k-1, t) e^{t} \mathrm{~d} t=\frac{e^{a}}{k-1} \gamma(k, a)$. \\
\hline (b) & $\int_{0}^{a} x^{k-1} e^{-\frac{x}{b}} \gamma\left(k, \frac{a-x}{c}\right) \mathrm{d} x= \begin{cases}\Gamma(k)^{2} b^{k} \frac{\gamma\left(2 k, \frac{a}{b}\right)}{\Gamma(2 k)}, & \text { if } b=c, \\
\Gamma(k)^{2}\left(\frac{b^{k} \gamma\left(k, \frac{a}{b}\right)}{\Gamma(k)}-a^{k} e^{-\frac{a}{c}} \sum_{j=0}^{k-1} \frac{\left(\frac{a}{c}\right)_{1}^{j} F_{1}\left(k ; k+j+1 ; a\left(\frac{1}{c}-\frac{1}{b}\right)\right)}{\Gamma(k+j+1)}\right), & \text { if } b \neq c .\end{cases}$ \\
\hline (c) & 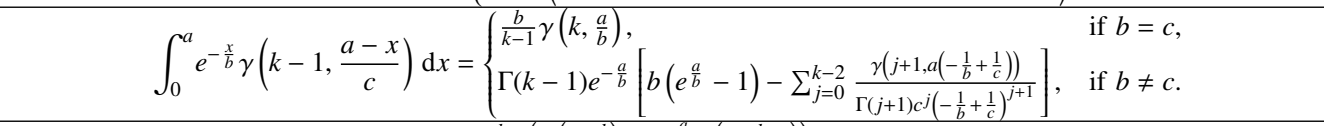 \\
\hline (d) & $\int_{0}^{a} e^{-\frac{x}{b}} \gamma\left(k-1, \frac{d-x}{c}\right) \mathrm{d} x \underset{a<d}{=} \begin{cases}\frac{b}{k-1}\left(\gamma\left(k, \frac{d}{b}\right)-e^{-\frac{a}{b}} \gamma\left(k, \frac{d-a}{b}\right)\right), & \text { if } b=c, \\
\Gamma(k-1)\left[b\left(1-e^{-\frac{a}{b}}\right)-e^{-\frac{d}{b}} \sum_{j=0}^{k-2} \frac{1}{\Gamma(j+1) c^{j}\left(-\frac{1}{b}+\frac{1}{c}\right)^{j+1}}\right. & \\
\left.\times\left(\gamma\left(j+1, d\left(-\frac{1}{b}+\frac{1}{c}\right)\right)-\gamma\left(j+1,(d-a)\left(-\frac{1}{b}+\frac{1}{c}\right)\right)\right)\right], & \text { if } b \neq c .\end{cases}$ \\
\hline (e) & $\int_{0}^{a} x^{k-1} e^{-\frac{x}{b}} \gamma\left(k, \frac{d-x}{c}\right) \mathrm{d} x \underset{a<d}{=} \begin{cases}\Gamma(k) b^{k}\left(\frac{\Gamma(k)}{\Gamma(2 k)} \gamma\left(2 k, \frac{d}{b}\right)-\gamma\left(k, \frac{d}{b}\right)+\gamma\left(k, \frac{a}{b}\right)\right) \\
+\Gamma(k) a^{k-1} e^{-\frac{d}{b}} \sum_{j=0}^{k-1} \frac{(d-a)^{j+1}}{\Gamma(j+2) b j} F_{1}\left(1,-k+1 ; j+2 ;-\frac{d-a}{a}\right), & \text { if } b=c, \\
\Gamma(k)\left[b^{k} \gamma\left(k, \frac{a}{b}\right)-e^{-\frac{d}{c}} \sum_{j=0}^{k-1} \frac{1}{\Gamma(j+1) c^{j}} \sum_{l=0}^{j}\left(\begin{array}{l}j \\
l\end{array}\right) \frac{d^{j-l}(-1) l}{\left(\frac{1}{b}-\frac{1}{c}\right)^{k+1}} \gamma\left(k+l, a\left(\frac{1}{b}-\frac{1}{c}\right)\right)\right], & \text { if } b \neq c .\end{cases}$ \\
\hline (f) & $\int_{0}^{a} x^{k_{1}-1} e^{-\frac{x}{b}} \Gamma\left(k_{2}, \frac{a-x}{c}\right) \mathrm{d} x= \begin{cases}\Gamma\left(k_{1}\right) \Gamma\left(k_{2}\right) b^{k_{1}}\left[\frac{\Gamma\left(k_{1}+k_{2}, \frac{a}{b}\right)}{\Gamma\left(k_{1}+k_{2}\right)}-\frac{\Gamma\left(k_{1}, \frac{a}{b}\right)}{\Gamma\left(k_{1}\right)}\right], & \text { if } b=c, \\
\Gamma\left(k_{1}\right) \Gamma\left(k_{2}\right) a^{k_{1}} e^{-\frac{a}{c}} \sum_{j=0}^{k_{2}-1} \frac{\left(\frac{a}{c}\right)_{1}^{j} F_{1}\left(k_{1} ; k_{1}+j+1 ; a\left(\frac{1}{c}-\frac{1}{b}\right)\right)}{\Gamma\left(k_{1}+j+1\right)}, & \text { if } b \neq c .\end{cases}$ \\
\hline (g) & $\int_{a}^{\infty} x^{k_{1}-1} e^{-\frac{x}{b}} \Gamma\left(k_{2}, \frac{x}{c}\right) \mathrm{d} x=\Gamma\left(k_{2}\right) \sum_{j=0}^{k_{2}-1} \frac{\Gamma\left(k_{1}+j, a\left(\frac{1}{b}+\frac{1}{c}\right)\right)}{c^{j}\left(\frac{1}{b}+\frac{1}{c}\right)^{k_{1}+j} \Gamma(j+1)}$ \\
\hline (h) & $\int_{a}^{d} x^{k_{1}-1} e^{-\frac{x}{b}} \Gamma\left(k_{2}, \frac{d-x}{b}\right) \mathrm{d} x=\Gamma\left(k_{1}\right) \Gamma\left(k_{2}\right) e^{-\frac{a}{b}} b^{k_{1}}\left[\frac{\Gamma\left(k_{1}+k_{2}, \frac{d-a}{b}\right)}{\Gamma\left(k_{1}+k_{2}\right)}-\frac{\Gamma\left(k_{1}, \frac{d-a}{b}\right)}{\Gamma\left(k_{1}\right)}\right]$ \\
\hline (i) & $\int_{a}^{\infty} x^{k_{1}-1} e^{-\frac{x}{b}} \Gamma\left(k_{2}, \frac{x-a}{c}\right) \mathrm{d} x=\Gamma\left(k_{2}\right) e^{\frac{a}{c}} \sum_{j=0}^{k_{2}-1} \frac{1}{c^{j} \Gamma(j+1)} \sum_{n=0}^{j}\left(\begin{array}{l}j \\
n\end{array}\right)(-a)^{j-n} \frac{\Gamma\left(k_{1}+n, a\left(\frac{1}{b}+\frac{1}{c}\right)\right)}{\left(\frac{1}{b}+\frac{1}{c}\right)^{k_{1}+n}}$. \\
\hline
\end{tabular}

